\title{
Vector-valued Hausdorff-Young inequality on compact groups
}

\author{
J. García-Cuerva and J.PARCet
}

\section{Introduction}

If $1 \leq p \leq 2$ and $p^{\prime}=p /(p-1)$ denotes the conjugate exponent of $p$, the classical Hausdorff-Young inequality establishes the boundedness of the Fourier transform from $L^{p}(\mathbb{R})$ into $L^{p^{\prime}}(\mathbb{R})$. Its proof is obtained by complex interpolation between the obvious case $p=1$ and the case $p=2$ given by Plancherel theorem. In the same spirit, Kunze applied in 1958 new techniques of non-commutative integration introduced by Dixmier [6] and Segal [23, 24] to study this inequality on locally compact unimodular groups, see [15]. In particular, for a compact non necessarily abelian group $G$, he proved the boundedness of the Fourier transform from $L^{p}(G)$ to $\mathcal{L}^{p^{\prime}}(\widehat{G})$. That is,

$$
\left(\sum_{\pi \in \widehat{G}} d_{\pi}\|\widehat{f}(\pi)\|_{S_{d_{\pi}}^{p^{\prime}}}^{p^{\prime}}\right)^{1 / p^{\prime}} \leq\left(\int_{G}|f(g)|^{p} d \mu(g)\right)^{1 / p} \quad \text { for } 1<p \leq 2,
$$

and with the obvious modifications for $p=1$. Here $\pi \in \widehat{G}$ denotes an irreducible unitary representation of $G, d_{\pi}$ is the degree of $\pi, S_{n}^{p^{\prime}}$ stands for the $n \times n$ dimensional Schatten class of exponent $p^{\prime}$ and $\mu$ is the Haar measure of $G$ normalized so that $\mu(G)=1$. On the other hand, Peetre presented in 1969 the first work [19] analyzing the Hausdorff-Young inequality for Banach valued functions $f: \mathbb{R} \rightarrow B$. In this case, the validity of the inequality

2000 Mathematics Subject Classification: 43A77, 46L07.

Research supported in part by the TMR Network 'Harmonic Analysis'.

Research supported in part by Project BFM 2001/0189, Spain. 
for some fixed $p$ depends on the Banach space $B$. This leads to the notion of Fourier type of a Banach space with respect to a locally compact abelian group, introduced by Milman in [17. The theory of Fourier type with respect to locally compact abelian groups was further developed by several authors, see [1], [3], 4], 10], 11] and [14].

However, as far as we know, in the non-commutative setting there is no analogous theory of Fourier type described in the literature. Our aim is to fill this gap. Namely, to analyze the validity of Kunze's results for vector-valued functions. In this work we investigate the validity of the Hausdorff-Young inequality for vector-valued functions defined on a compact group. As can be seen throughout the paper, compactness is an essential assumption in many of the results we present here. For a non-commutative compact group $G$, the vector-valued Fourier transform must be defined for irreducible unitary representations $\pi \in \widehat{G}$ and its values are vector-valued matrices. Therefore, just to start talking about the Hausdorff-Young inequality, one has to be able to define norms for vector-valued matrices. By Ruan's theorem [22], this matricial structure leads us to consider an operator space structure on the vector space where we are taking values. It appears clear that, in order to develop a theory of Fourier type in this context, we shall need to take values in operator spaces rather than Banach spaces. This crucial point is obviously at the root of the notion of Fourier type.

To conclude, we would like to point out that the theory initiated in this paper has been further developed in [12] and [13. Roughly speaking, the paper 12 deals with the sharpness of Theorems 6.5 and 6.8 for compact semisimple Lie groups, see section 6 for more on this topic. On the other hand, the notions of Fourier type and cotype of an operator space with respect to a compact group are extended in [13 to the more general setting of type and cotype with respect to a 'quantized orthonormal system'. This contains, for instance, the non-commutative versions of Rademacher or Gauss type and cotype. All this is used in [13] to obtain an operator space version of Kwapień theorem [16] characterizing Hilbert spaces by means of vectorvalued orthogonal series.

The organization of the paper is as follows. In section 1 we recall the notions of operator space theory and vector-valued Schatten classes that will be used in the sequel. In section 2 we define the Fourier transform on compact groups for vector-valued functions. We also study the spaces $\mathcal{L}_{E}^{p}(\widehat{G})$, where the Fourier transform takes values. Some results for which we have not found any reference have been proved for completeness. In particular, Proposition 
2.4 and Corollary 2.5 are specially relevant since they show that the spaces $\mathcal{L}_{E}^{\infty}(\widehat{G})$ and $\mathcal{L}_{E}^{1}(\widehat{G})$ behave with respect to the minimal and projective tensor products as the classical $L_{E}^{\infty}$ and $L_{E}^{1}$ do with respect to Grothendieck's tensor norms. Sections 3 and 4 are mainly devoted to showing that the notions of Fourier type and cotype are well defined, and also to prove some basic properties. Section 4 is specially far from the commutative theory since, as we show there, when dealing with abelian groups the notion of Fourier cotype reduces to the notion of Fourier type with respect to the dual group. In section [5, given an operator space $E$, we investigate the Fourier type and cotype of some general operator spaces related to $E$ such as subspaces, duals, interpolated spaces, etc... Finally, in section [6 we investigate the main examples, that is, Lebesgue spaces and Schatten classes. In particular, for the vector-valued ones we prove some quantized Minkowski inequalities that we shall need.

Acknowledgment. We thank Gilles Pisier for some useful comments.

\section{Operator spaces and Schatten classes}

The basic theories behind this paper are the theory of operator spaces and the subsequent theory of vector-valued Schatten classes. The reader is referred to [8] and 21] for a basic background on these topics and their connection with the present work. We begin with a brief summary of the results of operator space theory that will be used in the sequel.

(a) Definition of operator space. We will denote by $\mathcal{B}(\mathcal{H})$ the space of bounded linear operators on some Hilbert space $\mathcal{H}$. For our purposes an operator space $E$ can be defined as a closed subspace of $\mathcal{B}(\mathcal{H})$. Given an operator space $E \subset \mathcal{B}(\mathcal{H})$ we write $M_{n}(E)$ for the space $M_{n} \otimes E$ of $n \times n$ matrices with entries in $E$ and with the norm imposed by the natural embedding of $M_{n} \otimes E$ into $\mathcal{B}\left(l_{\mathcal{H}}^{2}(n)\right)$. Here $l_{\mathcal{H}}^{2}(n)$ denotes the Hilbert space of all $\mathcal{H}$-valued $n$-tuples with its natural inner product. On the other hand, given a vector space $E$ and a collection of norms $\|-\|_{n}$ on the spaces $M_{n} \otimes E$, one can impose some extra conditions to obtain what is called an operator space matrix norm or operator space structure on $E$, see Chapter 2 of [8]. One of the main results of the theory is the abstract characterization of operator spaces given by Ruan in [22]. Ruan's theorem can be rephrased by saying that for 
any operator space structure on a vector space $E$, there exists a unique Hilbert space $\mathcal{H}$ such that the norm from the operator space structure on $M_{n} \otimes E$ coincides with the norm induced by the space $\mathcal{B}\left(l_{\mathcal{H}}^{2}(n)\right)$.

(b) Complete boundedness. A linear mapping between operator spaces $u: E_{1} \rightarrow E_{2}$ is said to be completely bounded if the family of maps $I_{M_{n}} \otimes u: M_{n}\left(E_{1}\right) \rightarrow M_{n}\left(E_{2}\right)$ satisfy

$$
\|u\|_{c b}=\sup _{n \geq 1}\left\|I_{M_{n}} \otimes u\right\|_{\mathcal{B}\left(M_{n}\left(E_{1}\right), M_{n}\left(E_{2}\right)\right)}<\infty .
$$

We write $c b\left(E_{1}, E_{2}\right)$ for the Banach space of completely bounded maps from $E_{1}$ to $E_{2}$ with the $c b$ norm. Let $u \in c b\left(E_{1}, E_{2}\right)$, we say that $u$ is a complete isometry if the mappings $I_{M_{n}} \otimes u$ are isometries for all $n \geq 1$. Similarly $u$ is called completely contractive if $\|u\|_{c b} \leq 1$. We also say that $u$ is a complete isomorphism if it is a completely bounded linear isomorphism whose inverse is also completely bounded. Finally $u$ is a completely isometric isomorphism if it is also a complete isometry.

(c) Duality. Ruan's theorem was used by Blecher and Paulsen in [2] and by Effros and Ruan in [7] to get a duality theory in the category of operator spaces. It was shown that, by imposing on $M_{n} \otimes c b\left(E_{1}, E_{2}\right)$ the norm induced by $c b\left(E_{1}, M_{n}\left(E_{2}\right)\right)$, we obtain an operator space structure on $c b\left(E_{1}, E_{2}\right)$. In particular we have an operator space structure on the dual space $E^{\star}=c b(E, \mathbb{C})$. This notion of duality behaves as Banach space duality in many senses. For instance, it can be proved that the natural isometric inclusion $E \subset E^{\star \star}$ is a complete isometry.

(d) Tensor products. We are interested in two tensor norms that will be used repeatedly in this paper. Given two operator spaces $E_{1} \subset \mathcal{B}\left(\mathcal{H}_{1}\right)$ and $E_{2} \subset \mathcal{B}\left(\mathcal{H}_{2}\right)$ we define their minimal tensor product $E_{1} \otimes_{\min } E_{2}$ by the natural embedding of $E_{1} \otimes E_{2}$ into $\mathcal{B}\left(\mathcal{H}_{1} \otimes_{2} \mathcal{H}_{2}\right)$, where $\otimes_{2}$ stands for the Hilbertian tensor product. The minimal tensor product plays the role of the injective tensor product of Banach spaces in the category of operator spaces. Similarly, there exists an analog for operator spaces of the projective tensor product. It is denoted by $E_{1} \otimes^{\wedge} E_{2}$ and it was introduced in [2] and [7] independently. The tensor products $\otimes_{\min }$ and $\otimes^{\wedge}$ are associative and commutative. Here are some other properties 
which we will use in the sequel with no further reference

$$
\begin{array}{lll}
E_{1} \otimes^{\wedge} E_{2} & \rightarrow E_{1} \otimes_{\min } E_{2} & \text { is a comp. contraction } \\
E_{1}^{\star} \otimes_{\min } E_{2} & \rightarrow c b\left(E_{1}, E_{2}\right) & \text { is a comp. isometry } \\
E_{1} \otimes_{\min } E_{2} \rightarrow c b\left(E_{1}^{\star}, E_{2}\right) & \text { is a comp. isometry } \\
\left(E_{1} \otimes^{\wedge} E_{2}\right)^{\star} \rightarrow c b\left(E_{1}, E_{2}^{\star}\right) & \text { is a comp. isometric isomorph. }
\end{array}
$$

(e) Complex interpolation. Let $\left\{E_{0}, E_{1}\right\}$ be a compatible couple of Banach spaces in the sense of complex interpolation. Let us suppose that $E_{0}$ and $E_{1}$ have an operator space structure. In [20] Pisier showed that, if $E_{\theta}$ denotes the interpolation space $\left[E_{0}, E_{1}\right]_{\theta}$, one can define an operator space structure on $E_{\theta}$ by imposing on $M_{n} \otimes E_{\theta}$ the norm of the Banach space $\left[M_{n}\left(E_{0}\right), M_{n}\left(E_{1}\right)\right]_{\theta}$. He also proved the analog for operator spaces of the classical interpolation result for Banach spaces. Namely, if we assume that $u: E_{0}+E_{1} \rightarrow F_{0}+F_{1}$ satisfies the inequalities $\|u\|_{c b\left(E_{0}, F_{0}\right)} \leq C_{0}$ and $\|u\|_{c b\left(E_{1}, F_{1}\right)} \leq C_{1}$, then for $0<\theta<1$ we have the estimate

$$
\|u\|_{c b\left(E_{\theta}, F_{\theta}\right)} \leq C_{0}^{1-\theta} C_{1}^{\theta} .
$$

We now recall the definition and the main properties of the Schatten classes. The non-commutative analog of the $n$-dimensional Lebesgue space $l^{p}(n)$ is the Schatten class $S_{n}^{p}$ which is defined as the space $M_{n}$ of $n \times n$ complex matrices with the norm given by

(a) $\|A\|_{S_{n}^{p}}=\left(\operatorname{tr}|A|^{p}\right)^{1 / p}$, if $1 \leq p<\infty$.

(b) $\|A\|_{S_{n}^{\infty}}=\sup \left\{\|A x\|_{l^{2}(n)}:\|x\|_{\ell^{2}(n)} \leq 1\right\}$, if $p=\infty$.

Now we present the vector valued Schatten classes $S_{n}^{p}(E)$, introduced by Pisier in 21]. The point here is that the space $E$ where we take values has to be an operator space. If $p=\infty$, we have by definition $S_{n}^{\infty}=\mathcal{B}\left(l^{2}(n)\right)$ and so we obtain a natural operator space structure for $S_{n}^{\infty}$. We define $S_{n}^{\infty}(E)$ as the operator space $S_{n}^{\infty} \otimes_{\min } E$. It is obvious that $M_{n}(E)$ and $S_{n}^{\infty}(E)$ coincide, in what follows we shall write $S_{n}^{\infty}(E)$ for $M_{n}(E)$. If $p=1$, the duality $S_{n}^{1}=\left(S_{n}^{\infty}\right)^{\star}$ gives a natural operator space structure on $S_{n}^{1}$. We set $S_{n}^{1}(E)=S_{n}^{1} \otimes^{\wedge} E$. Finally, since the identity mapping $S_{n}^{1}(E) \rightarrow S_{n}^{\infty}(E)$ is contractive, we define the classes $S_{n}^{p}(E)$ by means of complex interpolation. Namely, $S_{n}^{p}(E)=\left[S_{n}^{\infty}(E), S_{n}^{1}(E)\right]_{1 / p}$. The next theorem summarizes some properties of the vector-valued Schatten classes that will be used repeatedly throughout the paper, see Chapter 1 of [21]. 
Remark 1.1 In the same fashion, it is possible to define a natural operator space structure on the Bochner-Lebesgue spaces, see Chapter 2 of [21].

Theorem 1.2 (Pisier) The vector-valued Schatten classes satisfy the following properties.

1. The cb norm. Let $E_{1}$ and $E_{2}$ be operator spaces and let $1 \leq p \leq \infty$. Then the cb norm of any linear mapping $u: E_{1} \rightarrow E_{2}$ is given by

$$
\|u\|_{c b}=\sup _{n \geq 1}\left\|I_{M_{n}} \otimes u\right\|_{\mathcal{B}\left(S_{n}^{p}\left(E_{1}\right), S_{n}^{p}\left(E_{2}\right)\right)} .
$$

2. Duality. Let $1 \leq p \leq \infty$ and let $p^{\prime}$ denote the conjugate exponent of p. The map $A \in S_{n}^{p^{\prime}}\left(E^{\star}\right) \mapsto \operatorname{tr}(A \cdot) \in S_{n}^{p}(E)^{\star}$ is completely isometric.

3. Complex interpolation. Let $1 \leq p_{0}, p_{1} \leq \infty, 0<\theta<1$ and assume that $\left\{E_{0}, E_{1}\right\}$ is a compatible couple of operator spaces. Then, letting $p_{\theta}^{-1}=(1-\theta) p_{0}^{-1}+\theta p_{1}^{-1}$, we have

$$
\left[S_{n}^{p_{0}}\left(E_{0}\right), S_{n}^{p_{1}}\left(E_{1}\right)\right]_{\theta}=S_{n}^{p_{\theta}}\left(E_{\theta}\right) .
$$

4. Ordered norms. Let $1 \leq p_{1} \leq p_{2} \leq \infty$. Then the identity map $S_{n}^{p_{1}}(E) \rightarrow S_{n}^{p_{2}}(E)$ is a contraction.

5. Fubini type theorems. Let $1 \leq p \leq \infty$ and let $n_{1}, n_{2}, n \geq 1$. Then, we have completely isometrically

$$
S_{n_{1}}^{p}\left(S_{n_{2}}^{p}(E)\right) \simeq S_{n_{2}}^{p}\left(S_{n_{1}}^{p}(E)\right) \quad \text { and } \quad S_{n}^{p}\left(L_{E}^{p}(\Omega)\right) \simeq L_{S_{n}^{p}(E)}^{p}(\Omega)
$$

\section{Vector-valued Fourier transform}

We assume the reader is familiar with the language of non-commutative abstract harmonic analysis on compact groups. In any case all the results we use here can be found in 9]. In what follows we shall assume that $G$ is a compact Hausdorff topological group endowed with its Haar measure $\mu$ normalized so that $\mu(G)=1$. The mapping $\pi: G \rightarrow U\left(\mathbb{C}^{d_{\pi}}\right)$ will denote an irreducible unitary representation of $G$ of degree $d_{\pi}$. That is, $\pi \in \widehat{G}$ where the symbol $\widehat{G}$ stands for the dual object of $G$. 
Definition 2.1 Given an operator space $E, f \in L_{E}^{1}(G)$ and $\pi \in \widehat{G}$, the vector-valued Fourier coefficient of $f$ at $\pi$ is defined as the operator

$$
\widehat{f}(\pi)=\int_{G} f(g) \pi(g)^{\star} d \mu(g) \in \mathcal{B}\left(\mathbb{C}^{d_{\pi}}, E^{d_{\pi}}\right) .
$$

We interpret this operator-valued integral in the weak sense. That is, given an orthonormal basis $\left\{v_{1}, v_{2}, \ldots, v_{d_{\pi}}\right\}$ of $\mathbb{C}^{d_{\pi}}$ and $u \in \mathbb{C}^{d_{\pi}}$, we define the $j$-th component of $\widehat{f}(\pi) u$, with respect to that basis, by the following element of $E$

$$
\int_{G} f(g)\left\langle\pi(g)^{\star} u, v_{j}\right\rangle d \mu(g) .
$$

Since $\pi(g)$ is unitary, it follows that the vector-valued Fourier coefficients are well-defined for all $f$ in $L_{E}^{1}(G)$. Once we have fixed the basis of $\mathbb{C}^{d_{\pi}}$, we can identify $\mathcal{B}\left(\mathbb{C}^{d_{\pi}}, E^{d_{\pi}}\right)$ with the space $M_{d_{\pi}} \otimes E$. This leads us to write the Fourier transform operator $\mathcal{F}_{G, E}$, in the form

$$
\mathcal{F}_{G, E}: L_{E}^{1}(G) \longrightarrow \prod_{\pi \in \widehat{G}} M_{d_{\pi}} \otimes E .
$$

The first step to study the Hausdorff-Young inequality is to find a natural $L^{p}$ norm for this Cartesian product, which we denote by $\mathcal{M}_{E}(\widehat{G})$.

Definition 2.2 Let $E$ be an operator space and $1 \leq p<\infty$, the spaces $\mathcal{L}_{E}^{p}(\widehat{G})$ are defined as follows

$$
\begin{aligned}
\mathcal{L}_{E}^{p}(\widehat{G}) & =\left\{A \in \mathcal{M}_{E}(\widehat{G}):\|A\|_{\mathcal{L}_{E}^{p}(\widehat{G})}=\left(\sum_{\pi \in \widehat{G}} d_{\pi}\left\|A^{\pi}\right\|_{S_{d_{\pi}}^{p}(E)}^{p}\right)^{1 / p}<\infty\right\} \\
\mathcal{L}_{E}^{\infty}(\widehat{G}) & =\left\{A \in \mathcal{M}_{E}(\widehat{G}):\|A\|_{\mathcal{L}_{E}^{\infty}(\widehat{G})}=\sup _{\pi \in \widehat{G}}\left\|A^{\pi}\right\|_{S_{d_{\pi}}^{\infty}(E)}<\infty\right\} .
\end{aligned}
$$

We write $\mathcal{L}^{p}(\widehat{G})$ for the case $E=\mathbb{C}$. Note that we require the vector space $E$ to be an operator space. This condition is necessary since we are making use of the spaces $S_{n}^{p}(E)$, see Pisier's monograph [21] for more on this topic. The family of spaces $\mathcal{L}_{E}^{p}(\widehat{G})$ is a particular case of a bigger family of spaces studied in Chapter 2 of [21], the spaces $\ell_{p}\left(\mu,\left\{E_{i}\right\}\right)$. This remark allows us to provide the spaces $\mathcal{L}_{E}^{p}(\widehat{G})$ with the natural operator space structure induced by $\ell_{p}\left(\mu,\left\{E_{i}\right\}\right)$. We now summarize the main properties of these spaces. 
(a) Duality. Let $1 \leq p<\infty$ and let $p^{\prime}$ be the conjugate exponent of $p$. Then the following map is a completely isometric isomorphism

$$
A \in \mathcal{L}_{E^{\star}}^{p^{\prime}}(\widehat{G}) \longmapsto \sum_{\pi \in \widehat{G}} d_{\pi} \operatorname{tr}\left(A^{\pi} \cdot\right) \in \mathcal{L}_{E}^{p}(\widehat{G})^{\star} .
$$

(b) Complex interpolation. Let $1 \leq p_{0}, p_{1} \leq \infty$. Assume that $\left\{E_{0}, E_{1}\right\}$ is a compatible couple of operator spaces. Then $\left\{\mathcal{L}_{E_{0}}^{p_{0}}(\widehat{G}), \mathcal{L}_{E_{1}}^{p_{1}}(\widehat{G})\right\}$ is also a compatible couple and, for $0<\theta<1$ and $p_{\theta}^{-1}=(1-\theta) p_{0}^{-1}+\theta p_{1}^{-1}$, we have that

$$
\left[\mathcal{L}_{E_{0}}^{p_{0}}(\widehat{G}), \mathcal{L}_{E_{1}}^{p_{1}}(\widehat{G})\right]_{\theta}=\mathcal{L}_{E_{\theta}}^{p_{\theta}}(\widehat{G}) .
$$

(c) Ordered norms. The embedding $\mathcal{L}_{E}^{p_{1}}(\widehat{G}) \rightarrow \mathcal{L}_{E}^{p_{2}}(\widehat{G})$ is contractive whenever $1 \leq p_{1} \leq p_{2} \leq \infty$.

(d) Fubini type theorems. Let $1 \leq p \leq \infty$ and let $n \geq 1$. Then the following are completely isometric isomorphisms

$$
S_{n}^{p}\left(\mathcal{L}_{E}^{p}(\widehat{G})\right) \simeq \mathcal{L}_{S_{n}^{p}(E)}^{p}(\widehat{G}) \quad \text { and } \quad \mathcal{L}_{L_{E}^{p}(\Omega)}^{p}(\widehat{G}) \simeq L_{\mathcal{L}_{E}^{p}(\widehat{G})}^{p}(\Omega) .
$$

We now present a couple of results concerning these spaces for which we have not found any reference. These will be especially useful in the study of the Fourier cotype, see for instance the proof of Proposition 4.3. We first need a technical result, which is an inequality of Hölder type.

Lemma 2.3 Let $E$ be an operator space, $n_{1}, n_{2} \geq 1$ and $1 \leq p \leq \infty$. Let us consider $A \in M_{n_{1}} \otimes E$ and $B_{i j} \in M_{n_{1}}$ for $1 \leq i, j \leq n_{2}$. Then

$$
\left\|\left(\operatorname{tr}\left(A B_{i j}\right)\right)\right\|_{S_{n_{2}}^{1}(E)} \leq\|A\|_{S_{n_{1}}^{p^{\prime}}(E)}\left\|\left(B_{i j}\right)\right\|_{S_{n_{1}}^{p}\left(S_{n_{2}}^{1}\right)} .
$$

Proof. If $a_{k l}$ and $b_{k l}^{i j}$ denote the entries of $A$ and $B_{i j}$ respectively, then we can write

$$
\left(\operatorname{tr}\left(A B_{i j}\right)\right)=\sum_{k, l=1}^{n_{1}}\left(b_{l k}^{i j}\right) \otimes a_{k l} \in M_{n_{2}} \otimes E .
$$


Hence, recalling the completely isometric isomorphism from $c b\left(S_{n}^{1}, E^{\star}\right)$ onto $\left(S_{n}^{1} \otimes^{\wedge} E\right)^{\star}$ given by $\Psi(A \otimes e)=\Phi(A)(e)$, we obtain

$$
\begin{aligned}
\left\|\left(\operatorname{tr}\left(A B_{i j}\right)\right)\right\|_{S_{n_{2}}^{1}(E)} & =\sup _{\|\Phi\|_{c b\left(S_{n_{2}}^{1}, E^{\star}\right)} \leq 1}\left|\sum_{k, l=1}^{n_{1}}\left[\Phi\left(b_{l k}^{i j}\right)\right]\left(a_{k l}\right)\right| \\
& =\sup _{\|\Phi\|_{c b\left(S_{n_{2}}^{1}, E^{\star}\right)} \leq 1}|\operatorname{tr}(C A)| \\
& \leq\|A\|_{S_{n_{1}}^{p^{\prime}}(E)} \sup _{\|\Phi\|_{c b\left(S_{n_{2}}^{1}, E^{\star}\right)} \leq 1}\|C\|_{S_{n_{1}}^{p}\left(E^{\star}\right)} \\
& \leq\|A\|_{S_{n_{1}}^{p^{\prime}}(E)}\left\|\left(B_{i j}\right)\right\|_{S_{n_{1}}^{p}\left(S_{n_{2}}^{1}\right)}
\end{aligned}
$$

where $C=\left[I_{M_{n_{1}}} \otimes \Phi\right]\left(B_{i j}\right) \in M_{n_{1}} \otimes E^{\star}$. This completes the proof.

For fixed $\pi_{0} \in \widehat{G}$ and $1 \leq i_{0}, j_{0} \leq d_{\pi_{0}}$, we define $M\left(\pi_{0}, i_{0}, j_{0}\right) \in \mathcal{M}_{\mathbb{C}}(\widehat{G})$ by the relations $M\left(\pi_{0}, i_{0}, j_{0}\right)_{i j}^{\pi}=\delta_{\pi \pi_{0}} \delta_{i i_{0}} \delta_{j j_{0}}$.

Proposition 2.4 The following is a completely isometric isomorphism

$$
\begin{aligned}
\Lambda: \mathcal{L}_{E}^{\infty}(\widehat{G}) & \longrightarrow c b\left(\mathcal{L}^{1}(\widehat{G}), E\right) \\
A & \longmapsto \sum_{\pi \in \widehat{G}} d_{\pi} \operatorname{tr}\left(A^{\pi} .\right)
\end{aligned}
$$

Proof. We just need to show that $\Lambda$ is an isometric isomorphism, since we have the natural isometric isomorphisms

$$
S_{n}^{\infty}\left(\mathcal{L}_{E}^{\infty}(\widehat{G})\right) \sim \mathcal{L}_{S_{n}^{\infty}(E)}^{\infty}(\widehat{G}) \quad \text { and } \quad c b\left(\mathcal{L}^{1}(\widehat{G}), S_{n}^{\infty}(E)\right) \sim S_{n}^{\infty}\left(c b\left(\mathcal{L}^{1}(\widehat{G}), E\right)\right) .
$$

1. $\Lambda$ is a contraction. By expressing the $c b$ norm in terms of the Schatten class $S^{1}$, we have

$$
\begin{aligned}
& \|\Lambda(A)\|_{c b\left(\mathcal{L}^{1}(\widehat{G}), E\right)} \\
& \quad \leq \sup _{n \geq 1}\left\{\sum_{\pi \in \widehat{G}} d_{\pi}\left\|\left(\operatorname{tr}\left(A^{\pi} B_{i j}^{\pi}\right)\right)\right\|_{S_{n}^{1}(E)}:\left\|\left(B_{i j}\right)\right\|_{S_{n}^{1}\left(\mathcal{L}^{1}(\widehat{G})\right)} \leq 1\right\}
\end{aligned}
$$

But Lemma 2.3 with $p=1$ gives

$$
\begin{aligned}
\sum_{\pi \in \widehat{G}} d_{\pi}\left\|\left(\operatorname{tr}\left(A^{\pi} B_{i j}^{\pi}\right)\right)\right\|_{S_{n}^{1}(E)} & \leq \sum_{\pi \in \widehat{G}} d_{\pi}\left\|A^{\pi}\right\|_{S_{d_{\pi}}^{\infty}(E)}\left\|\left(B_{i j}^{\pi}\right)\right\|_{S_{d_{\pi}^{1}}^{1}} \\
& \leq\|A\|_{\mathcal{L}_{E}^{\infty}(\widehat{G})}\left\|\left(B_{i j}\right)\right\|_{S_{n}^{1}\left(\mathcal{L}^{1}(\widehat{G})\right)}
\end{aligned}
$$


2. $\Lambda$ is an isometry. For fixed $\pi \in \widehat{G}$, we define $B(\pi, i, j)$ to be the element of $\mathcal{L}^{1}(\widehat{G})$ given by $d_{\pi}^{-1} M(\pi, j, i)$ and we denote by $\mathbf{B}(\pi)$ the matrix with entries $B(\pi, i, j)$ where $1 \leq i, j \leq d_{\pi}$. Due to the natural complete isometry $\mathcal{L}^{1}(\widehat{G}) \rightarrow c b\left(\mathcal{L}^{\infty}(\widehat{G}), \mathbb{C}\right)$, it is not difficult to check that $\|\mathbf{B}(\pi)\|_{S_{d_{\pi}}^{\infty}\left(\mathcal{L}^{1}(\widehat{G})\right)}=1$. Since this works for any $\pi \in \widehat{G}$, we get

$$
\|\Lambda(A)\|_{c b\left(\mathcal{L}^{1}(\widehat{G}), E\right)} \geq \sup _{\pi \in \widehat{G}}\left\|\left[I_{M_{d_{\pi}}} \otimes \Lambda(A)\right](\mathbf{B}(\pi))\right\|_{S_{d_{\pi}}^{\infty}(E)}=\|A\|_{\mathcal{L}_{E}^{\infty}(\widehat{G})} .
$$

3. $\Lambda$ is surjective. Let $\Phi \in c b\left(\mathcal{L}^{1}(\widehat{G}), E\right)$, then we define $A \in \mathcal{M}_{E}(\widehat{G})$ by the relation

$$
A^{\pi}=\frac{1}{d_{\pi}}(\Phi(M(\pi, j, i))), \quad \pi \in \widehat{G} .
$$

The definition of $A$ gives rise to the following expression

$$
\Phi(B)=\sum_{\pi \in \widehat{G}} \sum_{i, j=1}^{d_{\pi}} b_{i j}^{\pi} \Phi(M(\pi, i, j))=\sum_{\pi \in \widehat{G}} d_{\pi} \operatorname{tr}\left(A^{\pi} B^{\pi}\right)
$$

where $b_{i j}^{\pi}$ are the entries of $B^{\pi}$. Therefore it suffices to check that $A \in \mathcal{L}_{E}^{\infty}(\widehat{G})$. But following the notation of Step 2, we obtain

$$
\begin{aligned}
\left\|A^{\pi}\right\|_{S_{d_{\pi}}^{\infty}(E)} & =\frac{1}{d_{\pi}}\|(\Phi(M(\pi, j, i)))\|_{S_{d_{\pi}}^{\infty}(E)} \\
& \leq\|\Phi\|_{c b\left(\mathcal{L}^{1}(\widehat{G}), E\right)}\|\mathbf{B}(\pi)\|_{S_{d_{\pi}}^{\infty}\left(\mathcal{L}^{1}(\widehat{G})\right)} \leq\|\Phi\|_{c b\left(\mathcal{L}^{1}(\widehat{G}), E\right)} .
\end{aligned}
$$

Since $\Phi \in \operatorname{cb}\left(\mathcal{L}^{1}(\widehat{G}), E\right)$, we have a uniform upper bound.

The space $\mathcal{L}_{E}^{\infty}(\widehat{G})$ behaves with respect to the minimal tensor product as $L_{E}^{\infty}(\Omega)$ does with respect to the injective tensor product in the category of Banach spaces. Namely, as a consequence of Proposition [2.4, we have that $\mathcal{L}^{\infty}(\widehat{G}) \otimes_{\min } E \hookrightarrow \mathcal{L}_{E}^{\infty}(\widehat{G})$ is a complete isometry . The space $\mathcal{L}_{E}^{1}(\widehat{G})$ behaves in the same fashion with respect to the projective tensor product.

Corollary 2.5 The identity $\mathcal{L}^{1}(\widehat{G}) \otimes^{\wedge} E \rightarrow \mathcal{L}_{E}^{1}(\widehat{G})$ is completely isometric.

Proof. By Proposition 3.2.2 of [8] it suffices to check that the adjoint mapping is a complete isometric isomorphism. But Proposition 2.4 gives the following chain $\mathcal{L}_{E}^{1}(\widehat{G})^{\star} \simeq \mathcal{L}_{E^{\star}}^{\infty}(\widehat{G}) \simeq c b\left(\mathcal{L}^{1}(\widehat{G}), E^{\star}\right) \simeq\left(\mathcal{L}^{1}(\widehat{G}) \otimes^{\wedge} E\right)^{\star}$ of completely isometric isomorphisms. This completes the proof. 
For the sake of completeness we introduce the space $C_{0}(\widehat{G}, E)$. It is defined as the collection of those $A \in \mathcal{L}_{E}^{\infty}(\widehat{G})$ satisfying

$\forall \varepsilon>0$ we have $\left\|A^{\pi}\right\|_{S_{d_{\pi}}^{\infty}(E)}<\varepsilon$ except for finitely many $\pi \in \widehat{G}$.

As a subspace of $\mathcal{L}_{E}^{\infty}(\widehat{G})$ this space inherits a natural operator space structure. The only two results about the spaces $\mathcal{L}_{E}^{p}(\widehat{G})$ that fail at $p=\infty$ are the density of $\mathcal{L}^{p}(\widehat{G}) \otimes E$ in $\mathcal{L}_{E}^{p}(\widehat{G})$ and duality, the predual of $\mathcal{L}_{E^{\star}}^{1}(\widehat{G})$ is not $\mathcal{L}_{E}^{\infty}(\widehat{G})$. However, it is easy to see the density of $C_{0}(\widehat{G}) \otimes E$ in $C_{0}(\widehat{G}, E)$. On the other hand, the dual of $C_{0}(\widehat{G}, E)$ is completely isomorphic to $\mathcal{L}_{E^{\star}}^{1}(\widehat{G})$.

Proposition 2.6 The following is a completely isometric isomorphism

$$
\begin{aligned}
\Lambda: \mathcal{L}_{E^{\star}}^{1}(\widehat{G}) & \longrightarrow C_{0}(\widehat{G}, E)^{\star} \\
A & \longmapsto \sum_{\pi \in \widehat{G}} d_{\pi} \operatorname{tr}\left(A^{\pi} \cdot\right)
\end{aligned}
$$

Proof. Taking into account the natural isometric isomorphisms given by $S_{n}^{1}\left(\mathcal{L}_{E^{\star}}^{1}(\widehat{G})\right) \sim \mathcal{L}_{S_{n}^{\infty}(E)^{\star}}^{1}(\widehat{G})$ and $C_{0}\left(\widehat{G}, S_{n}^{\infty}(E)\right)^{\star} \sim S_{n}^{1}\left(C_{0}(\widehat{G}, E)^{\star}\right)$ it is enough to see that $\Lambda$ is an isometric isomorphism. We prove this fact in several steps.

1. $\Lambda$ is a contraction. This is an obvious consequence of the duality action on the Schatten classes $S_{n}^{p}(E)$

$$
\begin{aligned}
\|\Lambda(A)\|_{C_{0}(\widehat{G}, E)^{\star}} & \leq \sup _{\|B\|_{C_{0}(\widehat{G}, E)} \leq 1} \sum_{\pi \in \widehat{G}} d_{\pi}\left|\operatorname{tr}\left(A^{\pi} B^{\pi}\right)\right| \\
& \leq \sup _{\|B\|_{C_{0}(\widehat{G}, E)} \leq 1} \sum_{\pi \in \widehat{G}} d_{\pi}\left\|A^{\pi}\right\|_{S_{d_{\pi}}^{1}\left(E^{\star}\right)}\left\|B^{\pi}\right\|_{S_{d_{\pi}}^{\infty}(E)} \\
& \leq\|A\|_{\mathcal{L}_{E^{\star}}^{1}(\widehat{G})} .
\end{aligned}
$$

2. $\Lambda$ is an isometry. Let $A \in \mathcal{L}_{E^{\star}}^{1}(\widehat{G})$. For all $\varepsilon>0$ there exists a finite set $I_{A, \varepsilon} \subset \widehat{G}$ such that

$$
\sum_{\pi \notin I_{A, \varepsilon}} d_{\pi}\left\|A^{\pi}\right\|_{S_{d_{\pi}}^{1}\left(E^{\star}\right)}<\varepsilon / 2 .
$$

Furthermore, for all $\pi \in \widehat{G}$ there exists $B_{\varepsilon}^{\pi} \in S_{d_{\pi}}^{\infty}(E)$ of norm 1 such that

$$
\operatorname{tr}\left(A^{\pi} B_{\varepsilon}^{\pi}\right)>\left\|A^{\pi}\right\|_{S_{d_{\pi}}^{1}\left(E^{\star}\right)}-\frac{\varepsilon / 2}{\left|I_{A, \varepsilon}\right| \max _{\pi \in I_{A, \varepsilon}} d_{\pi}}
$$


where $\left|I_{A, \varepsilon}\right|$ denotes the number of elements of $I_{A, \varepsilon}$. Let $C_{\varepsilon}$ be the element of $C_{0}(\widehat{G}, E)$ of norm 1 defined by $C_{\varepsilon}^{\pi}=B_{\varepsilon}^{\pi}$ if $\pi \in I_{A, \varepsilon}$ and 0 otherwise. This Step is completed by taking $\varepsilon$ arbitrarily small in the expression

$$
\|\Lambda(A)\|_{C_{0}(\widehat{G}, E)^{\star}} \geq\left|\sum_{\pi \in \widehat{G}} d_{\pi} \operatorname{tr}\left(A^{\pi} C_{\varepsilon}^{\pi}\right)\right|>\|A\|_{\mathcal{L}_{E^{\star}}^{1}(\widehat{G})}-\varepsilon .
$$

3. $\Lambda$ is surjective. Let $\Phi \in C_{0}(\widehat{G}, E)^{\star}$. Then we define $A \in \mathcal{M}_{E^{\star}}(\widehat{G})$ by the relation

$$
A^{\pi}=\frac{1}{d_{\pi}}(\Phi(M(\pi, j, i) \otimes \cdot)), \quad \pi \in \widehat{G} .
$$

As in Proposition [2.4, it can be shown that $\Phi=\Lambda(A)$ with $A \in \mathcal{L}_{E^{\star}}^{1}(\widehat{G})$.

\section{$3 \quad$ Fourier type}

Let $E$ be an operator space and let $1 \leq p \leq 2$. Given $f \in L^{p}(G)$ and $e \in E$ it is obvious that the Fourier transform of $f \otimes e$ coincides with $\widehat{f} \otimes e$. Thus, the Hausdorff-Young inequality for compact groups (see [15] or Lemma 5.1 below) provides the relation $\mathcal{F}_{G, E}\left(L^{p}(G) \otimes E\right) \subset \mathcal{L}^{p^{\prime}}(\widehat{G}) \otimes E$. This motivates the following definition.

Definition 3.1 Let $1 \leq p \leq 2$ and let $p^{\prime}$ be the conjugate exponent of $p$. We say that the operator space $E$ has Fourier type $p$ with respect to the compact group $G$ if the Fourier transform $\mathcal{F}_{G, E}: L^{p}(G) \otimes E \rightarrow \mathcal{L}^{p^{\prime}}(\widehat{G}) \otimes E$ can be extended to a completely bounded operator

$$
\Lambda_{G, E, p}^{1}: L_{E}^{p}(G) \longrightarrow \mathcal{L}_{E}^{p^{\prime}}(\widehat{G}) .
$$

In that case, we shall denote by $\mathcal{C}_{p}^{1}(E, G)$ the $c b$ norm of $\Lambda_{G, E, p}^{1}$.

Remark 3.2 If the compact group $G$ is also abelian, there exists already a notion (introduced by Milman in [17]) of Fourier type of a Banach space with respect to $G$. The only difference with Milman's notion is that here we require the extended operator to be completely bounded while in the commutative setting, only the boundedness of this operator is required.

The first natural question that arises after the definition of Fourier type is if the extension of $\mathcal{F}_{G, E}$ is always the natural one. That is, let us suppose that the operator space $E$ has Fourier type $p$ with respect to $G$. Then we wonder if $\Lambda_{G, E, p}^{1}(f)=\mathcal{F}_{G, E}(f)$ for all $f \in L_{E}^{p}(G)$. 
Lemma 3.3 $\|\widehat{f}\|_{\mathcal{L}_{E}^{\infty}(\widehat{G})} \leq\|f\|_{L_{E}^{1}(G)}$ for all $f \in L_{E}^{1}(G)$.

Proof. Since $E$ is an operator space we have $E \subset \mathcal{B}(\mathcal{H})$ for some Hilbert space $\mathcal{H}$. Hence, if $\mathbf{h}=\left(h_{1}, h_{2}, \ldots, h_{d_{\pi}}\right) \in \ell_{\mathcal{H}}^{2}\left(d_{\pi}\right)$, we can write

$$
\begin{aligned}
\|\widehat{f}(\pi)\|_{S_{d_{\pi}}^{\infty}(E)} & =\|\widehat{f}(\pi)\|_{\mathcal{B}\left(\ell_{\mathcal{H}}^{2}\left(d_{\pi}\right)\right)} \\
& \leq \sup _{\|\mathbf{h}\|_{\ell_{\mathcal{H}}^{2}\left(d_{\pi}\right)} \leq 1}\left(\sum_{i=1}^{d_{\pi}}\left[\int_{G}\left\|f(g)\left(\sum_{j=1}^{d_{\pi}} \overline{\pi_{j i}(g)} h_{j}\right)\right\|_{\mathcal{H}} d \mu(g)\right]^{2}\right)^{1 / 2} .
\end{aligned}
$$

Applying Minkowski inequality for integrals we get

$$
\|\widehat{f}(\pi)\|_{S_{d_{\pi}}^{\infty}(E)} \leq \sup _{\|\mathbf{h}\|_{\ell_{\mathcal{H}}^{2}\left(d_{\pi}\right)} \leq 1} \int_{G}\|f(g)\|_{E}\left(\sum_{i=1}^{d_{\pi}}\left\|\sum_{j=1}^{d_{\pi}} \overline{\pi_{j i}(g)} h_{j}\right\|_{\mathcal{H}}^{2}\right)^{1 / 2} d \mu(g) .
$$

Therefore we just need to check the inequality

$$
\sup _{\|\mathbf{h}\|_{\ell_{\mathcal{H}}^{2}\left(d_{\pi}\right)} \leq 1}\left(\sum_{i=1}^{d_{\pi}}\left\|\sum_{j=1}^{d_{\pi}} \overline{\pi_{j i}(g)} h_{j}\right\|_{\mathcal{H}}^{2}\right)^{1 / 2} \leq 1
$$

for all $\pi \in \widehat{G}$ and almost all $g \in G$. But this is a simple consequence of the unitarity of $\pi(g)$ for any $g \in G$. This completes the proof.

Proposition 3.4 Let $E$ be an operator space having Fourier type $p$ with respect to $G$. Then $\Lambda_{G, E, p}^{1}(f)=\mathcal{F}_{G, E}(f)$ for all $f \in L_{E}^{p}(G)$.

Proof. Let $\left\{f_{n}\right\}_{n=1}^{\infty} \subset L^{p}(G) \otimes E$ be a sequence convergent to $f$ in the norm of $L_{E}^{p}(G)$. Then, applying Lemma 3.3, we have

$$
\begin{aligned}
& \left\|\Lambda_{G, E, p}^{1}(f)-\mathcal{F}_{G, E}(f)\right\|_{\mathcal{L}_{E}^{\infty}(\widehat{G})} \\
& \quad \leq\left\|\Lambda_{G, E, p}^{1}\left(f-f_{n}\right)\right\|_{\mathcal{L}_{E}^{\infty}(\widehat{G})}+\left\|\mathcal{F}_{G, E}\left(f_{n}-f\right)\right\|_{\mathcal{L}_{E}^{\infty}(\widehat{G})} \\
& \quad \leq\left\|\Lambda_{G, E, p}^{1}\left(f-f_{n}\right)\right\|_{\mathcal{L}_{E}^{p^{\prime}(\widehat{G})}}+\left\|\mathcal{F}_{G, E}\left(f_{n}-f\right)\right\|_{\mathcal{L}_{E}^{\infty}(\widehat{G})} \\
& \quad \leq \mathcal{C}_{p}^{1}(E, G)\left\|f-f_{n}\right\|_{L_{E}^{p}(G)}+\left\|f_{n}-f\right\|_{L_{E}^{1}(G)} \\
& \quad \leq\left(\mathcal{C}_{p}^{1}(E, G)+1\right)\left\|f-f_{n}\right\|_{L_{E}^{p}(G)}
\end{aligned}
$$

The result follows by taking the limit in $n$. This completes the proof.

As it is well-known, every Banach space has Fourier type 1 in the sense of Milman [17. In the following result, which extends Lemma 3.3, we show that every operator space has Fourier type 1. 
Proposition 3.5 We have $\mathcal{C}_{1}^{1}(E, G)=1$ for every pair $(E, G)$.

Proof. Let us denote by $\max B$ the operator space which results when we impose on the Banach space $B$ its max quantization, see Chapter 3 of $[8$ for the details. Let $E_{1}$ and $E_{2}$ be operator spaces. Then the natural identification $c b\left((\max B) \otimes^{\wedge} E_{1}, E_{2}\right) \simeq \mathcal{B}\left(B, c b\left(E_{1}, E_{2}\right)\right)$, given by $\Psi\left(b \otimes e_{1}\right)=\Phi(b)\left(e_{1}\right)$, is a completely isomorphic isomorphism. This follows by the factorization

$$
c b\left((\max B) \otimes^{\wedge} E_{1}, E_{2}\right) \simeq c b\left(\max B, c b\left(E_{1}, E_{2}\right)\right) \simeq \mathcal{B}\left(B, c b\left(E_{1}, E_{2}\right)\right)
$$

which is composed of completely isometric isomorphisms, see Chapters 3 and 7 of [8]. Therefore, since the space $L_{E}^{1}(G)$ can be rewritten as $\max L^{1}(G) \otimes^{\wedge} E$, we get that

$$
\mathcal{C}_{1}^{1}(E, G)=\sup _{\|f\|_{L^{1}(G)} \leq 1}\|\widehat{f} \otimes \cdot\|_{c b\left(E, \mathcal{L}_{E}^{\infty}(\widehat{G})\right)} \leq \sup _{\|f\|_{L^{1}(G)} \leq 1}\|\widehat{f}\|_{\mathcal{L}^{\infty}(\widehat{G})}=1
$$

by the Hausdorff-Young inequality on compact groups, see [15] or Lemma 5.1 below. Recall that the supremum is attained taking $f$ to be the constant function 1. This completes the proof.

Remark 3.6 There exists an alternative approach to this result using similar arguments to those employed in the proof of Proposition 4.3.

The following corollary exhibits the Fourier type as a stronger condition on the pair $(E, G)$ as the exponent $p$ approaches 2 . Its proof follows by means of Proposition 3.5 and the complex interpolation method.

Corollary 3.7 Let $1 \leq p_{1} \leq p_{2} \leq 2$ and assume that the operator space $E$ has Fourier type $p_{2}$ with respect to $G$. Then $E$ has Fourier type $p_{1}$ with respect to $G$. Moreover we have $\mathcal{C}_{p_{1}}^{1}(E, G) \leq \mathcal{C}_{p_{2}}^{1}(E, G)^{p_{2}^{\prime}} / p_{1}^{\prime}$.

A vector-valued version of the Riemann-Lebesgue lemma on compact groups follows easily from Proposition 3.5 and the scalar result.

Corollary 3.8 $\mathcal{F}_{G, E}\left(L_{E}^{1}(G)\right) \subset C_{0}(\widehat{G}, E)$ for every operator space $E$. 


\section{Fourier cotype}

If $G$ is a locally compact abelian group, the Fourier inversion theorem asserts that any $f \in L^{1}(G)$ such that $\widehat{f} \in L^{1}(\widehat{G})$ can be recovered as

$$
f(g)=\widehat{\widehat{f}}\left(g^{-1}\right) \quad \text { for almost every } g \in G .
$$

Furthermore, if $G$ is compact one can conclude that the operators $\mathcal{F}_{G}^{-1}$ and $\mathcal{F}_{\widehat{G}}$ are essentially the same via the topological isomorphism from $G$ onto its bidual, given by the Pontrjagin duality theorem. In the vector-valued context this means that, in order to study the operator $\mathcal{F}_{G, E}^{-1}$, it suffices to study the Fourier transform $\mathcal{F}_{\widehat{G}, E}$. For this reason we do not find the concept of Fourier cotype in the commutative theory. However, for a non-commutative compact group $G$, the Fourier inversion theorem and the Pontrjagin duality theorem are no longer valid since the dual object $\widehat{G}$ is not even a group. These considerations explain why the study of the inverse operator $\mathcal{F}_{G, E}^{-1}$ should not be a trivial consequence of the analysis of the operator $\mathcal{F}_{G, E}$.

Let $E$ be an operator space and $1 \leq p \leq 2$. Arguing as in section 3, we can deduce the relation $\mathcal{F}_{G, E}^{-1}\left(\mathcal{L}^{p}(\widehat{G}) \otimes E\right) \subset L^{p^{\prime}}(G) \otimes E$. This follows from Kunze's result for the inverse Fourier transform on compact groups (see [15] or Lemma 5.1 below). This motivates the following definition.

Definition 4.1 Let $1 \leq p \leq 2$ and let $p^{\prime}$ be the conjugate exponent of $p$. We say that the operator space $E$ has Fourier cotype $p^{\prime}$ with respect to the compact group $G$ if the operator $\mathcal{F}_{G, E}^{-1}: \mathcal{L}^{p}(\widehat{G}) \otimes E \rightarrow L^{p^{\prime}}(G) \otimes E$ can be extended to a completely bounded operator

$$
\Lambda_{G, E, p^{\prime}}^{2}: \mathcal{L}_{E}^{p}(\widehat{G}) \longrightarrow L_{E}^{p^{\prime}}(G) .
$$

In that case, we shall denote by $\mathcal{C}_{p^{\prime}}^{2}(E, G)$ the $c b$ norm of $\Lambda_{G, E, p^{\prime}}^{2}$.

Remark 4.2 Now it is obvious that, for compact abelian groups, the notion of Fourier cotype is the completely bounded version of Milman's notion of Fourier type with respect to the dual group $\widehat{G}$.

Plancherel theorem for compact groups gives an explicit formula for the action of $\mathcal{F}_{G}^{-1}$ on $\mathcal{L}^{2}(\widehat{G})$ and, by the natural embeddings, also on $\mathcal{L}^{p}(\widehat{G})$ for $1 \leq p \leq 2$. It is obvious that this formula remains valid if we take tensor 
products. Namely, given $1 \leq p \leq 2$, the action of the operator $\mathcal{F}_{G, E}^{-1}$ on $\mathcal{L}^{p}(\widehat{G}) \otimes E$ is given by

$$
A \in \mathcal{L}^{p}(\widehat{G}) \otimes E \longmapsto \sum_{\pi \in \widehat{G}} d_{\pi} \operatorname{tr}\left(A^{\pi} \pi(\cdot)\right) \in L^{p^{\prime}}(G) \otimes E .
$$

Therefore, if we want our definition of Fourier cotype to be natural, we need affirmative answers for the following questions.

(a) Does the operator $\Lambda_{G, E, p^{\prime}}^{2}$ preserve the given explicit formula? That is, if the operator space $E$ has Fourier cotype $p^{\prime}$ with respect to $G$, we ask whether for all $A \in \mathcal{L}_{E}^{p}(\widehat{G})$ we have

$$
\Lambda_{G, E, p^{\prime}}^{2}(A)=\sum_{\pi \in \widehat{G}} d_{\pi} \operatorname{tr}\left(A^{\pi} \pi(\cdot)\right)
$$

If $A \in \mathcal{L}_{E}^{p}(\widehat{G})$, it has a countable support $I_{A}=\left\{\pi_{k}\right\}_{k=1}^{\infty} \subset \widehat{G}$. Then we define $A_{n} \in \mathcal{L}^{p}(\widehat{G}) \otimes E$ by the relations $A_{n}^{\pi}=A^{\pi}$ if $\pi=\pi_{k}$ for $1 \leq k \leq n$ and $A_{n}^{\pi}=0$ otherwise. Denoting by

$$
f=\sum_{\pi \in \widehat{G}} d_{\pi} \operatorname{tr}\left(A^{\pi} \pi(\cdot)\right) \quad \text { and } \quad f_{n}=\sum_{\pi \in \widehat{G}} d_{\pi} \operatorname{tr}\left(A_{n}^{\pi} \pi(\cdot)\right)
$$

we obtain that

$$
\begin{aligned}
\left\|\Lambda_{G, E, p^{\prime}}^{2}(A)-f\right\|_{L_{E}^{p^{\prime}}(G)} & \leq\left\|\Lambda_{G, E, p^{\prime}}^{2}\left(A-A_{n}\right)\right\|_{L_{E}^{p^{\prime}}(G)}+\left\|f-f_{n}\right\|_{L_{E}^{p^{\prime}}(G)} \\
& \leq C_{p^{\prime}}^{2}(E, G)\left\|A-A_{n}\right\|_{\mathcal{L}_{E}^{p}(\widehat{G})}+\left\|f-f_{n}\right\|_{L_{E}^{p^{\prime}}(G)}
\end{aligned}
$$

The first term of the sum is arbitrarily small as $n$ tends to infinity. For the second term it is not difficult to check that the sequence $\left\{f_{n}\right\}_{n=1}^{\infty}$ is Cauchy. Thus, replacing this sequence if necessary by an appropriate subsequence, we can assume $\left\|f_{n_{2}}-f_{n_{1}}\right\|_{L_{E}^{p^{\prime}}(G)}<2^{-m}$ for all $n_{1}, n_{2} \geq m$. Hence

$$
\left\|f-f_{n}\right\|_{L_{E}^{p^{\prime}(G)}} \leq \sum_{k=n+1}^{\infty}\left\|f_{k}-f_{k-1}\right\|_{L_{E}^{p^{\prime}(G)}}<\sum_{k=n}^{\infty} \frac{1}{2^{k}}
$$

and $\Lambda_{G, E, p^{\prime}}^{2}(A)=\sum_{\pi \in \widehat{G}} d_{\pi} \operatorname{tr}\left(A^{\pi} \pi(\cdot)\right)$ as we wanted. 
(b) Does the operator $\Lambda_{G, E, p^{\prime}}^{2}$ coincide with the inverse of the vector-valued Fourier transform? That is, if the operator space $E$ has Fourier cotype $p^{\prime}$ with respect to $G$, we ask whether for all $A \in \mathcal{L}_{E}^{p}(\widehat{G})$ we have

$$
\mathcal{F}_{G, E} \circ \Lambda_{G, E, p^{\prime}}^{2}(A)=A \text {. }
$$

Using the same notation as above we just need to see that $\widehat{f}=A$. Given $\pi \in \widehat{G}$ we take $n_{\pi}$ to be the smallest positive integer satisfying $\pi \neq \pi_{k}$ for $k \geq n_{\pi}$. Then it is obvious that $\widehat{f}(\pi)-A^{\pi}=\left(\widehat{f}-\widehat{f}_{n}\right)(\pi)$ for all $n \geq n_{\pi}$ and therefore it is enough to estimate the entries of that matrix. Namely,

$$
\begin{aligned}
\left\|\left(\left(\widehat{f}-\widehat{f}_{n}\right)(\pi)\right)_{i j}\right\|_{E} & \leq \int_{G}\left\|\left(f-f_{n}\right)(g)\right\|_{E}\left|\pi_{j i}(g)\right| d \mu(g) \\
& \leq\left\|f-f_{n}\right\|_{L_{E}^{p^{\prime}}(G)}\left\|\pi_{j i}\right\|_{L^{p}(G)} \\
& \leq \mathcal{C}_{p^{\prime}}^{2}(E, G)\left\|A-A_{n}\right\|_{\mathcal{L}_{E}^{p}(\widehat{G})}
\end{aligned}
$$

which is arbitrarily small for large $n$.

Proposition 4.3 We have $\mathcal{C}_{\infty}^{2}(E, G)=1$ for every pair $(E, G)$.

Proof. By property 1 of Theorem 1.2 and a density argument, we have to see that for all $n \geq 1$, any family of vectors $A_{i j} \in \mathcal{L}^{1}(\widehat{G}) \otimes E$ and almost all $g \in G$, we have

$$
\left\|\left(\sum_{\pi \in \widehat{G}} d_{\pi} \operatorname{tr}\left(A_{i j}^{\pi} \pi(g)\right)\right)\right\|_{S_{n}^{\infty}(E)} \leq\left\|\left(A_{i j}\right)\right\|_{S_{n}^{\infty}\left(\mathcal{L}_{E}^{1}(\widehat{G})\right)} .
$$

If we consider a vector $A \in \mathcal{L}_{E}^{1}(\widehat{G})$ as an element of $\operatorname{cb}\left(\mathcal{L}^{\infty}(\widehat{G}), E\right)$ by the relation

$$
B \in \mathcal{L}^{\infty}(\widehat{G}) \longmapsto \sum_{\pi \in \widehat{G}} d_{\pi} \operatorname{tr}\left(A^{\pi} B^{\pi}\right) \in E
$$

then it is easy to see that, for $B_{g} \in \mathcal{L}^{\infty}(\widehat{G})$ defined by $B_{g}^{\pi}=\pi(g)$, we have

$$
\begin{aligned}
\left\|\left(\sum_{\pi \in \widehat{G}} d_{\pi} \operatorname{tr}\left(A_{i j}^{\pi} \pi(g)\right)\right)\right\|_{S_{n}^{\infty}(E)} & =\left\|\left(\sum_{\pi \in \widehat{G}} d_{\pi} \operatorname{tr}\left(A_{i j}^{\pi} \cdot\right)\left(B_{g}\right)\right)\right\|_{S_{n}^{\infty}(E)} \\
& \leq\left\|\left(\sum_{\pi \in \widehat{G}} d_{\pi} \operatorname{tr}\left(A_{i j}^{\pi} \cdot\right)\right)\right\|_{c b\left(\mathcal{L}^{\infty}(\widehat{G}), S_{n}^{\infty}(E)\right)} \\
\leq & \left\|\left(A_{i j}\right)\right\|_{S_{n}^{\infty}\left(\mathcal{L}^{1}(\widehat{G}) \otimes^{\wedge} E\right)}
\end{aligned}
$$


where the last inequality follows from the complete contraction given by $\mathcal{L}^{1}(\widehat{G}) \otimes^{\wedge} E \rightarrow \mathcal{L}^{1}(\widehat{G}) \otimes_{\text {min }} E \rightarrow c b\left(\mathcal{L}^{\infty}(\widehat{G}), E\right)$. Finally, we get the desired relation by Corollary 2.5 . We have shown that $\mathcal{C}_{\infty}^{2}(E, G) \leq 1$. The reverse inequality follows from Corollary 5.3 below.

Corollary 4.4 Let $1 \leq p_{1} \leq p_{2} \leq 2$ and assume that the operator space $E$ has Fourier cotype $p_{2}^{\prime}$ with respect to $G$. Then $E$ has Fourier cotype $p_{1}^{\prime}$ with respect to $G$. Moreover we have $\mathcal{C}_{p_{1}^{\prime}}^{2}(E, G) \leq \mathcal{C}_{p_{2}^{\prime}}^{2}(E, G)^{p_{2}^{\prime} / p_{1}^{\prime}}$.

\section{Duality, $c b$ distance and some other topics}

Let $E$ be an operator space. The aim of this section is to study the Fourier type and cotype of some operator spaces related to $E$. We begin by stating the scalar-valued Hausdorff-Young inequality. Recall that we write $\check{A}$ to denote $\mathcal{F}_{G}^{-1}(A)$.

Lemma 5.1 (Hausdorff-Young inequality) Let $1 \leq p \leq 2$ and let $p^{\prime}$ be the conjugate exponent of $p$ :

1. If $f \in L^{p}(G)$, then $\widehat{f} \in \mathcal{L}^{p^{\prime}}(\widehat{G})$ and $\left\|\mathcal{F}_{G}\right\|_{c b\left(L^{p}(G), \mathcal{L}^{p^{\prime}}(\widehat{G})\right)}=1$.

2. If $A \in \mathcal{L}^{p}(\widehat{G})$, then $\check{A} \in L^{p^{\prime}}(G)$ and $\left\|\mathcal{F}_{G}^{-1}\right\|_{c b\left(\mathcal{L}^{p}(\widehat{G}), L^{p^{\prime}}(G)\right)}=1$.

Note that this statement of the inequality goes a bit further than Kunze's original result since we are asserting that the Fourier transform is not only bounded but completely bounded. The proof is straightforward, first one checks that $\mathcal{F}_{G}$ is a complete contraction from $L^{1}(G)$ into $\mathcal{L}^{\infty}(\widehat{G})$. But, since $L^{1}(G)$ is equipped with its max operator space structure, the $c b$ norm coincides with the operator norm, see Chapter 3 [8]. The same argument works to see that the inverse Fourier transform is a complete contraction from $\mathcal{L}^{1}(\widehat{G})$ to $L^{\infty}(G)$, now $L^{\infty}(G)$ is equipped with its min operator space structure. These facts can also be justified as simple consequences of Propositions 3.5 and 4.3. Second, from the Plancherel theorem for compact groups, it is easy to check that $\mathcal{F}_{G}$ is a complete isometric isomorphism from $L^{2}(G)$ onto $\mathcal{L}^{2}(\widehat{G})$. By complex interpolation the general case is obtained and, the fact that the $c b$ norm of the Fourier transform is not smaller than 1 for any $1 \leq p \leq 2$ can be checked by testing with the constant function 1 . 


\section{$5.1 \quad$ Basic results}

We begin by the simplest case. Namely, the Fourier type and cotype of the subspaces of $E$. The following result is a trivial consequence of property 1 of Theorem [1.2.

Proposition 5.2 Let $F$ be a closed subspace of $E$, then we have the estimates $\mathcal{C}_{p}^{1}(F, G) \leq \mathcal{C}_{p}^{1}(E, G)$ and $\mathcal{C}_{q^{\prime}}^{2}(F, G) \leq \mathcal{C}_{q^{\prime}}^{2}(E, G)$ for any $1 \leq p, q \leq 2$.

Corollary $5.3 \mathcal{C}_{p}^{1}(E, G) \geq 1$ and $\mathcal{C}_{q^{\prime}}^{2}(E, G) \geq 1$ for any $1 \leq p, q \leq 2$.

Now we consider complex interpolation of operator spaces. The proof of the next result is also straightforward.

Proposition 5.4 Let $1 \leq p_{0}, p_{1} \leq 2$ and assume that $\left\{E_{0}, E_{1}\right\}$ is compatible for complex interpolation. Then $\mathcal{C}_{p_{\theta}}^{1}\left(E_{\theta}, G\right) \leq \mathcal{C}_{p_{0}}^{1}\left(E_{0}, G\right)^{1-\theta} \mathcal{C}_{p_{1}}^{1}\left(E_{1}, G\right)^{\theta}$ for $p_{\theta}^{-1}=(1-\theta) p_{0}^{-1}+\theta p_{1}^{-1}$. A similar result holds for the Fourier cotype.

\subsection{Duality}

The following theorem can be rephrased by saying that Fourier type and cotype are dual notions.

Theorem 5.5 Let $E$ be an operator space, $1 \leq p \leq 2$ and $p^{\prime}$ its conjugate exponent. Then

1. E has Fourier type $p$ with respect to a compact group $G$ if and only if $E^{\star}$ has Fourier cotype $p^{\prime}$ with respect to $G$.

2. E has Fourier cotype $p^{\prime}$ with respect to a compact group $G$ if and only if $E^{\star}$ has Fourier type $p$ with respect to $G$.

Moreover, we have $\mathcal{C}_{p}^{1}(E, G)=\mathcal{C}_{p^{\prime}}^{2}\left(E^{\star}, G\right)$ and $\mathcal{C}_{p}^{1}\left(E^{\star}, G\right)=\mathcal{C}_{p^{\prime}}^{2}(E, G)$.

Proof. We just prove the equality $\mathcal{C}_{p}^{1}(E, G)=\mathcal{C}_{p^{\prime}}^{2}\left(E^{\star}, G\right)$ since the proof of the second identity is essentially the same. The case $p=1$ follows from Propositions 3.5] and 4.3, thus we assume that $1<p \leq 2$. 
Step 1. $\mathcal{C}_{p}^{1}(E, G) \geq \mathcal{C}_{p^{\prime}}^{2}\left(E^{\star}, G\right)$. By a density argument and property 1 of Theorem 1.2 we just need to check that the following inequality holds

$$
\left\|\left(\sum_{\pi \in \widehat{G}} d_{\pi} \operatorname{tr}\left(A_{i j}^{\pi} \pi(\cdot)\right)\right)\right\|_{S_{n}^{p^{\prime}\left(L_{E^{\star}}^{p^{\prime}}(G)\right)}} \leq \mathcal{C}_{p}^{1}(E, G)\left\|\left(A_{i j}\right)\right\|_{S_{n}^{p^{\prime}}\left(\mathcal{L}_{E^{\star}}^{p}(\widehat{G})\right)}
$$

for any family $A_{i j} \in \mathcal{L}^{p}(\widehat{G}) \otimes E^{\star}(1 \leq i, j \leq n)$ and all $n \geq 1$. But we have the completely isometric isomorphism $S_{n}^{p^{\prime}}\left(L_{E^{\star}}^{p^{\prime}}(G)\right) \simeq L_{S_{n}^{p}(E)^{\star}}^{p^{\prime}}(G)$. So for all $\varepsilon>0$ there exists $f^{\varepsilon} \in L_{S_{n}^{p}(E)}^{p}(G)$ of norm 1 such that

$$
\begin{aligned}
& \left\|\left(\sum_{\pi \in \widehat{G}} d_{\pi} \operatorname{tr}\left(A_{i j}^{\pi} \pi(\cdot)\right)\right)\right\|_{S_{n}^{p^{\prime}}\left(L_{E^{\star}}^{p^{\prime}}(G)\right)} \\
& \leq(1+\varepsilon)\left|\int_{G} \operatorname{tr}\left[\left(\sum_{\pi \in \widehat{G}} d_{\pi} \operatorname{tr}\left(A_{i j}^{\pi} \pi(g)\right)\right)\left(f_{i j}^{\varepsilon}(g)\right)\right] d \mu(g)\right|
\end{aligned}
$$

and where $f_{i j}^{\varepsilon}$, the entries of $f^{\varepsilon}$, belong to $L^{p}(G) \otimes E$. If we denote by $\mathcal{I}$ the integral over $G$ written above, then we would like to prove that

$$
\mathcal{I}=\sum_{i, j=1}^{n} \sum_{\pi \in \widehat{G}} d_{\pi} \int_{G}\left\langle\operatorname{tr}\left(A_{i j}^{\pi} \pi(g)\right), f_{j i}^{\varepsilon}(g)\right\rangle d \mu(g) .
$$

Taking into account that $A_{i j} \in \mathcal{L}^{p}(\widehat{G}) \otimes E^{\star}$ and $f_{i j}^{\varepsilon} \in L^{p}(G) \otimes E$ it suffices to show that the expressions

$$
\begin{aligned}
& \mathcal{I}_{1}=\int_{G} \sum_{\pi \in \widehat{G}} d_{\pi} \operatorname{tr}\left(A^{\pi} \pi(g)\right) f(g) d \mu(g) \\
& \mathcal{I}_{2}=\sum_{\pi \in \widehat{G}} d_{\pi} \int_{G} \operatorname{tr}\left(A^{\pi} \pi(g)\right) f(g) d \mu(g)
\end{aligned}
$$

coincide for all $A \in \mathcal{L}^{p}(\widehat{G})$ and all $f \in L^{p}(G)$. But this is an easy computation that we leave to the reader. In summary we obtain

$$
\begin{aligned}
& \left\|\left(\sum_{\pi \in \widehat{G}} d_{\pi} \operatorname{tr}\left(A_{i j}^{\pi} \pi(\cdot)\right)\right)\right\|_{S_{n}^{p^{\prime}}\left(L_{E^{\star}}^{p^{\prime}}(G)\right)} \\
& \leq(1+\varepsilon)\left|\sum_{i, j=1}^{n} \sum_{\pi \in \widehat{G}} d_{\pi} \int_{G}\left\langle\operatorname{tr}\left(A_{i j}^{\pi} \pi(g)\right), f_{j i}^{\varepsilon}(g)\right\rangle d \mu(g)\right|
\end{aligned}
$$




$$
\begin{aligned}
& =(1+\varepsilon)\left|\sum_{i, j=1}^{n} \sum_{\pi \in \widehat{G}} d_{\pi} \operatorname{tr}\left(\left\langle A_{i j}^{\pi}, \widehat{\tau\left(f_{j i}^{\varepsilon}\right)}(\pi)\right\rangle\right)\right| \\
& =(1+\varepsilon)\left|\operatorname{tr}\left[\left(A_{i j}\right)\left(\widehat{\tau\left(f_{i j}^{\varepsilon}\right)}\right)\right]\right|
\end{aligned}
$$

where $\tau(f)(g)=f\left(g^{-1}\right)$. This step is concluded by the following inequality

$$
\begin{aligned}
& \left|\operatorname{tr}\left[\left(A_{i j}\right)\left(\widehat{\tau\left(f_{i j}^{\varepsilon}\right)}\right)\right]\right| \leq\left\|\left(A_{i j}\right)\right\|_{S_{n}^{p^{\prime}\left(\mathcal{L}_{E^{\star}}^{p}(\widehat{G})\right)}}\left\|\left(\widehat{\tau\left(f_{i j}^{\varepsilon}\right)}\right)\right\|_{S_{n}^{p}\left(\mathcal{L}_{E}^{p^{\prime}}(\widehat{G})\right)} \\
& \leq \mathcal{C}_{p}^{1}(E, G)\left\|\left(A_{i j}\right)\right\|_{S_{n}^{p^{\prime}}\left(\mathcal{L}_{E^{\star}}^{p}(\widehat{G})\right)}\left\|f^{\varepsilon}\right\|_{S_{n}^{p}\left(L_{E}^{p}(G)\right)} .
\end{aligned}
$$

Step 2. $\mathcal{C}_{p}^{1}(E, G) \leq \mathcal{C}_{p^{\prime}}^{2}\left(E^{\star}, G\right)$. By the same reasons given in Step 1, it suffices to check that

$$
\left\|\left(\widehat{f}_{i j}\right)\right\|_{S_{n}^{p^{\prime}}\left(\mathcal{L}_{E}^{p^{\prime}}(\widehat{G})\right)} \leq \mathcal{C}_{p^{\prime}}^{2}\left(E^{\star}, G\right)\left\|\left(f_{i j}\right)\right\|_{S_{n}^{p^{\prime}}\left(L_{E}^{p}(G)\right)}
$$

for any family $f_{i j} \in L^{p}(G) \otimes E(1 \leq i, j \leq n)$ and all $n \geq 1$. Given $\varepsilon>0$, the complete isometry

$$
S_{n}^{p^{\prime}}\left(\mathcal{L}_{E}^{p^{\prime}}(\widehat{G})\right) \simeq \mathcal{L}_{S_{n}^{p^{\prime}}(E)}^{p^{\prime}}(\widehat{G})
$$

provides the existence of $\mathbf{A}^{\varepsilon} \in \mathcal{L}_{S_{n}^{p}\left(E^{\star}\right)}^{p}(\widehat{G})$ of norm 1 such that

$$
\left\|\left(\widehat{f}_{i j}\right)\right\|_{S_{n}^{p^{\prime}}\left(\mathcal{L}_{E}^{p^{\prime}}(\widehat{G})\right)} \leq(1+\varepsilon)\left|\sum_{\pi \in \widehat{G}} d_{\pi} \operatorname{tr}\left[\left(A_{i j}^{\varepsilon, \pi}\right)\left(\widehat{f}_{i j}(\pi)\right)\right]\right|
$$

and where $A_{i j}^{\varepsilon}$, the entries of $\mathbf{A}^{\varepsilon}$, belong to $\mathcal{L}^{p}(\widehat{G}) \otimes E^{\star}$. If $\mathcal{S}$ denotes the sum written above, then we can argue as in Step 1 to obtain

$$
\mathcal{S}=\sum_{i, j=1}^{n} \int_{G}\left\langle\sum_{\pi \in \widehat{G}} d_{\pi} \operatorname{tr}\left(A_{i j}^{\varepsilon, \pi} \pi(g)^{\star}\right), f_{j i}(g)\right\rangle d \mu(g) .
$$

Therefore

$$
\begin{aligned}
\left\|\left(\widehat{f}_{i j}\right)\right\|_{S_{n}^{p^{\prime}}\left(\mathcal{L}_{E}^{p^{\prime}}(\widehat{G})\right)} & \leq(1+\varepsilon)\left|\sum_{i, j=1}^{n} \int_{G}\left\langle\mathcal{F}_{G, E^{\star}}^{-1}\left(A_{i j}^{\varepsilon}\right)(g), f_{j i}\left(g^{-1}\right)\right\rangle d \mu(g)\right| \\
& =(1+\varepsilon)\left|\operatorname{tr}\left[\left(\mathcal{F}_{G, E^{\star}}^{-1}\left(A_{i j}^{\varepsilon}\right)\right)\left(\tau\left(f_{i j}\right)\right)\right]\right|
\end{aligned}
$$




$$
\begin{aligned}
& \leq(1+\varepsilon)\left\|\mathcal{F}_{G, E^{\star}}^{-1}\left(\mathbf{A}^{\varepsilon}\right)\right\|_{S_{n}^{p}\left(L_{E}^{p}(G)^{\star}\right)}\left\|\left(f_{i j}\right)\right\|_{S_{n}^{p^{\prime}}\left(L_{E}^{p}(G)\right)} \\
& =(1+\varepsilon)\left\|\mathcal{F}_{G, E^{\star}}^{-1}\left(\mathbf{A}^{\varepsilon}\right)\right\|_{S_{n}^{p}\left(L_{E^{\star}}^{p^{\prime}}(G)\right)}\left\|\left(f_{i j}\right)\right\|_{S_{n}^{p^{\prime}}\left(L_{E}^{p}(G)\right)} \\
& \leq(1+\varepsilon) \mathcal{C}_{p^{\prime}}^{2}\left(E^{\star}, G\right)\left\|\left(f_{i j}\right)\right\|_{S_{n}^{p^{\prime}}\left(L_{E}^{p}(G)\right)}
\end{aligned}
$$

The proof is completed by taking $\varepsilon$ arbitrarily small.

Remark 5.6 There exists another possible approach to this result. Namely, $E$ has Fourier type $p$ if and only if the corresponding Fourier transform operator is completely bounded. But then, by Proposition 3.2.2 of [8], the adjoint operator is also completely bounded with the same $c b$ norm. Moreover, it can be checked that the adjoint coincides with the inverse of the Fourier transform for functions taking values in $E^{\star}$. This gives the first equality of Theorem 5.5. The second equality follows in a similar fashion.

Corollary $5.7 \mathcal{C}_{p}^{1}(E, G)=\mathcal{C}_{p}^{1}\left(E^{\star \star}, G\right)$ and $\mathcal{C}_{p^{\prime}}^{2}(E, G)=\mathcal{C}_{p^{\prime}}^{2}\left(E^{\star \star}, G\right)$.

\subsection{The $c b$ distance}

There exists a natural analog in the category of operator spaces of the Banach-Mazur distance, due to Pisier. It is called the $c b$ distance and it is defined by

$$
d_{c b}\left(E_{1}, E_{2}\right)=\inf \left\{\|u\|_{c b\left(E_{1}, E_{2}\right)}\left\|u^{-1}\right\|_{c b\left(E_{2}, E_{1}\right)}\right\}
$$

where the infimum runs over all complete isomorphisms $u: E_{1} \rightarrow E_{2}$.

Theorem 5.8 Let $E_{1}$ and $E_{2}$ be operator spaces and let $G$ be a compact group. Then the following inequalities hold for $1 \leq p \leq 2$

$$
\begin{aligned}
\mathcal{C}_{p}^{1}\left(E_{2}, G\right) & \leq d_{c b}\left(E_{1}, E_{2}\right) \mathcal{C}_{p}^{1}\left(E_{1}, G\right) \\
\mathcal{C}_{p^{\prime}}^{2}\left(E_{2}, G\right) & \leq d_{c b}\left(E_{1}, E_{2}\right) \mathcal{C}_{p^{\prime}}^{2}\left(E_{1}, G\right) \\
\mathcal{C}_{p}^{1}\left(E_{2}, G\right) & \leq d_{c b}\left(E_{1}, E_{2}^{\star}\right) \mathcal{C}_{p^{\prime}}^{2}\left(E_{1}, G\right) \\
\mathcal{C}_{p^{\prime}}^{2}\left(E_{2}, G\right) & \leq d_{c b}\left(E_{1}, E_{2}^{\star}\right) \mathcal{C}_{p}^{1}\left(E_{1}, G\right) .
\end{aligned}
$$


Proof. The last two inequalities follow from the first two ones plus duality. Let us assume that the first inequality holds, then the second inequality is also an immediate consequence of Theorem 5.5

$$
\begin{aligned}
\mathcal{C}_{p^{\prime}}^{2}\left(E_{2}, G\right) & =\mathcal{C}_{p}^{1}\left(E_{2}^{\star}, G\right) \leq d_{c b}\left(E_{1}^{\star}, E_{2}^{\star}\right) \mathcal{C}_{p}^{1}\left(E_{1}^{\star}, G\right) \\
& =d_{c b}\left(E_{1}^{\star}, E_{2}^{\star}\right) \mathcal{C}_{p^{\prime}}^{2}\left(E_{1}, G\right)=d_{c b}\left(E_{1}, E_{2}\right) \mathcal{C}_{p^{\prime}}^{2}\left(E_{1}, G\right)
\end{aligned}
$$

where we have applied the identity $\|u\|_{c b\left(E_{1}, E_{2}\right)}=\left\|u^{\star}\right\|_{c b\left(E_{2}^{\star}, E_{1}^{\star}\right)}$ to justify the equality $d_{c b}\left(E_{1}^{\star}, E_{2}^{\star}\right)=d_{c b}\left(E_{1}, E_{2}\right)$, see [8] for details. Therefore we will be done if we prove the validity of the first inequality. For that it suffices to see

$$
\left\|\left(\widehat{f}_{i j}\right)\right\|_{S_{n}^{p^{\prime}\left(\mathcal{L}_{E_{2}}^{p^{\prime}}(\widehat{G})\right)}} \leq\|u\|_{c b}\left\|u^{-1}\right\|_{c b} \mathcal{C}_{p}^{1}\left(E_{1}, G\right)\left\|\left(f_{i j}\right)\right\|_{S_{n}^{p^{\prime}\left(L_{E_{2}}^{p}(G)\right)}}
$$

for any family $f_{i j} \in L^{p}(G) \otimes E_{2}(1 \leq i, j \leq n)$, any complete isomorphism $u: E_{1} \rightarrow E_{2}$ and all $n \geq 1$. But

$$
\begin{aligned}
& \left\|\left(\widehat{f}_{i j}\right)\right\|_{S_{n}^{p^{\prime}}\left(\mathcal{L}_{E_{2}}^{p^{\prime}}(\widehat{G})\right)}=\left(\sum_{\pi \in \widehat{G}} d_{\pi}\left\|\left(\widehat{f}_{i j}(\pi)\right)\right\|_{S_{d_{n} n}^{p^{\prime}}\left(E_{2}\right)}^{p^{\prime}}\right)^{1 / p^{\prime}} \\
& \leq\|u\|_{c b}\left(\sum_{\pi \in \widehat{G}} d_{\pi}\left\|\left(I_{M_{d_{\pi} n}} \otimes u^{-1}\right)\left(\widehat{f}_{i j}(\pi)\right)\right\|_{S_{d_{\pi n}}^{p^{\prime}}\left(E_{1}\right)}^{p^{p^{\prime}}}\right)^{1 / p^{\prime}} \\
& \quad=\|u\|_{c b}\left\|\left(\mathcal{F}_{G, E_{1}}\left(\left(I_{L^{p}(G)} \otimes u^{-1}\right)\left(f_{i j}\right)\right)\right)\right\|_{\mathcal{L}_{S_{n}^{p^{\prime}}\left(E_{1}\right)}(\widehat{G})} \\
& \leq\|u\|_{c b} \mathcal{C}_{p}^{1}\left(E_{1}, G\right)\left\|\left(\left(I_{L^{p}(G)} \otimes u^{-1}\right)\left(f_{i j}\right)\right)\right\|_{S_{n}^{p^{\prime}}\left(L_{E_{1}}^{p}(G)\right)} \\
& \quad\|u\|_{c b} \mathcal{C}_{p}^{1}\left(E_{1}, G\right)\left\|I_{L^{p}(G)} \otimes u^{-1}\right\|_{c b}\left\|\left(f_{i j}\right)\right\|_{S_{n}^{p^{\prime}}\left(L_{E_{2}}^{p}(G)\right)} \\
& =\|u\|_{c b}\left\|u^{-1}\right\|_{c b} \mathcal{C}_{p}^{1}\left(E_{1}, G\right)\left\|\left(f_{i j}\right)\right\|_{S_{n}^{p^{\prime}\left(L_{E_{2}}^{p}(G)\right)}} .
\end{aligned}
$$

This completes the proof.

We recall here that, if $\mathcal{O S}_{n}$ denote the class of all $n$-dimensional operator spaces, Pisier proved the estimate $d_{c b}\left(E, O H_{n}\right) \leq \sqrt{n}$ for any operator space $E \in \mathcal{O S}_{n}$. Here $O H_{n}$ denotes the $n$-dimensional operator Hilbert space $O H$, see [20]. Therefore, by taking $E_{1}=l^{2}(n)$ in Theorem 5.8 and invoking the results of the next section, we get the following result.

Corollary 5.9 We have $\mathcal{C}_{2}^{1}(E, G), \mathcal{C}_{2}^{2}(E, G) \leq \sqrt{n}$ for any $E \in \mathcal{O S}_{n}$. 


\section{Basic examples}

We study here the Fourier type and cotype of Lebesgue spaces, Schatten classes and their vector-valued versions. We start with the statement of some inequalities of Minkowski type in the operator space setting.

If $1 \leq p_{1} \leq p_{2} \leq \infty$ and the measure spaces $\left(\Omega_{1}, \mathcal{M}_{1}, \nu_{1}\right),\left(\Omega_{2}, \mathcal{M}_{2}, \nu_{2}\right)$ are $\sigma$-finite, then the classical Minkowski inequality for integrals asserts that the natural map

$$
L_{L^{p_{2}\left(\Omega_{2}\right)}}^{p_{1}}\left(\Omega_{1}\right) \longrightarrow L_{L^{p_{1}\left(\Omega_{1}\right)}}^{p_{2}}\left(\Omega_{2}\right)
$$

is contractive. The same happens if our functions $f: \Omega_{1} \times \Omega_{2} \rightarrow E$ take values in a Banach space $E$. We are interested in the complete boundedness of this operator and some others in which we replace the Lebesgue spaces $L^{p}(\Omega)$ by the Schatten classes $S_{n}^{p}$. For this purpose, by complex interpolation, it suffices to check the cases $p_{1}=p_{2}$ and $\left(p_{1}, p_{2}\right)=(1, \infty)$. The first case follows from the Fubini type results stated in Theorem 1.2. The second case reduces to see that the natural map $E_{1} \otimes^{\wedge}\left(E_{2} \otimes_{\text {min }} E_{3}\right) \rightarrow\left(E_{1} \otimes^{\wedge} E_{2}\right) \otimes_{\text {min }} E_{3}$ is a complete contraction. The proof of this result can be found in Theorem 8.1.10 of [8]. In summary we can state the following results.

Theorem 6.1 (Quantized Minkowski inequalities) Let us consider an operator space $E$ and let $1 \leq p_{1} \leq p_{2} \leq \infty$.

1. Lebesgue spaces. Let $\left(\Omega_{1}, \mathcal{M}_{1}, \nu_{1}\right)$ and $\left(\Omega_{2}, \mathcal{M}_{2}, \nu_{2}\right)$ be $\sigma$-finite measure spaces. Then the following natural map is a complete contraction

$$
L_{L_{E}^{p_{2}}\left(\Omega_{2}\right)}^{p_{1}}\left(\Omega_{1}\right) \longrightarrow L_{L_{E}^{p_{1}}\left(\Omega_{1}\right)}^{p_{2}}\left(\Omega_{2}\right) .
$$

2. Schatten classes. Let $k_{1}, k_{2} \geq 1$, then the following natural map is a complete contraction

$$
S_{k_{1}}^{p_{1}}\left(S_{k_{2}}^{p_{2}}(E)\right) \longrightarrow S_{k_{2}}^{p_{2}}\left(S_{k_{1}}^{p_{1}}(E)\right) .
$$

3. Combined results. Let $(\Omega, \mathcal{M}, \nu)$ be a measure space and $k \geq 1$. Then the following natural maps are complete contractions

$$
S_{k}^{p_{1}}\left(L_{E}^{p_{2}}(\Omega)\right) \longrightarrow L_{S_{k}^{p_{1}}(E)}^{p_{2}}(\Omega) \quad \text { and } \quad L_{S_{k}^{p_{2}}(E)}^{p_{1}}(\Omega) \longrightarrow S_{k}^{p_{2}}\left(L_{E}^{p_{1}}(\Omega)\right) .
$$

Remark 6.2 The arguments sketched above in order to prove Theorem 6.1 need extra hypotheses. A different proof, without those unnecessary hypotheses, can be found in the Thesis [18] of the second-named author. 
In the study of the Fourier type of a Banach space with respect to a locally compact abelian group, Andersson [1] gave the following version of Minkowski inequality for regular measures. We recall that our notion of regular measure is the same as the one given in [5].

Proposition 6.3 (Andersson) Let $1 \leq p_{1} \leq p_{2}<\infty$ and assume that $\left(\Omega_{1}, \mathcal{M}_{1}, \nu_{1}\right)$ and $\left(\Omega_{2}, \mathcal{M}_{2}, \nu_{2}\right)$ are regular measure spaces. Let us denote by $H$ the space of functions $f: \Omega_{1} \times \Omega_{2} \rightarrow \mathbb{C}$ such that $|f|$ is bounded lower semicontinuous and $\left\|f_{\omega_{2}}\right\|_{L^{p_{1}\left(\Omega_{1}\right)}}$ is bounded in $\Omega_{2}$. Then the following natural map is contractive

$$
L_{L^{p_{2}\left(\Omega_{2}\right)}}^{p_{1}}\left(\Omega_{1}\right) \cap H \longrightarrow L_{L^{p_{1}\left(\Omega_{1}\right)}}^{p_{2}}\left(\Omega_{2}\right) \cap H .
$$

Let us note that, if $(\Omega, \mathcal{M}, \nu)$ denotes a regular measure space and we take $\Omega_{1}=G$ and $\Omega_{2}=\Omega$ in Proposition 6.3 then the space $C_{c}(G \times \Omega)$ of continuous functions with compact support, defined on $G \times \Omega$ and with values in $\mathbb{C}$, is contained in the space $H$. Hence, by the density of $C_{c}(G \times \Omega)$ in $L_{L^{p_{2}(\Omega)}}^{p_{1}}(G)$ and $L_{L^{p_{1}(G)}}^{p_{2}}(\Omega)$, we deduce that the natural map

$$
L_{L^{p_{2}(\Omega)}}^{p_{1}}(G) \longrightarrow L_{L^{p_{1}(G)}}^{p_{2}}(\Omega)
$$

is a contraction whenever $1 \leq p_{1} \leq p_{2}<\infty$. Then, by the same arguments that we gave in the proof of Theorem 6.1. we conclude that we have in fact a complete contraction. Furthermore, the same happens if we take $\Omega_{1}=\Omega$ and $\Omega_{2}=G$. Therefore we have shown the validity of the following result, which we enunciate for vector-valued functions since its proof is analogous.

Lemma 6.4 Let $1 \leq p_{1} \leq p_{2}<\infty$ and assume that $E$ is an operator space, $G$ is a compact group and $(\Omega, \mathcal{M}, \nu)$ is a regular measure space. Then the following natural maps are complete contractions

$$
L_{L_{E}^{p_{2}}(\Omega)}^{p_{1}}(G) \longrightarrow L_{L_{E}^{p_{1}}(G)}^{p_{2}}(\Omega) \quad \text { and } \quad L_{L_{E}^{p_{2}}(G)}^{p_{1}}(\Omega) \longrightarrow L_{L_{E}^{p_{1}}(\Omega)}^{p_{2}}(G) .
$$

Theorem 6.5 Let $1 \leq p, q \leq 2$ and assume that $E$ is an operator space having Fourier type $p$ and Fourier cotype $q^{\prime}$ with respect to a compact group $G$. Let $(\Omega, \mathcal{M}, \nu)$ be a regular or $\sigma$-finite measure space. Then

1. $L_{E}^{r}(\Omega)$ has Fourier type $p$ with respect to $G$ for all $p \leq r \leq p^{\prime}$.

2. $L_{E}^{s}(\Omega)$ has Fourier cotype $q^{\prime}$ with respect to $G$ for all $q \leq s \leq q^{\prime}$. 
Moreover, $\mathcal{C}_{p}^{1}\left(L_{E}^{r}(\Omega), G\right)=\mathcal{C}_{p}^{1}(E, G)$ and $\mathcal{C}_{q^{\prime}}^{2}\left(L_{E}^{s}(\Omega), G\right)=\mathcal{C}_{q^{\prime}}^{2}(E, G)$.

Proof. We start by proving the relation $\mathcal{C}_{p}^{1}\left(L_{E}^{r}(\Omega), G\right)=\mathcal{C}_{p}^{1}(E, G)$. For $p=1$ we just need to apply Proposition 3.5. Thus we assume that $1<p \leq 2$. The inequality $\mathcal{C}_{p}^{1}\left(L_{E}^{r}(\Omega), G\right) \geq \mathcal{C}_{p}^{1}(E, G)$ follows from Proposition 5.2 and, by complex interpolation, it then suffices to see that $\mathcal{C}_{p}^{1}\left(L_{E}^{r}(\Omega), G\right) \leq \mathcal{C}_{p}^{1}(E, G)$ for $r=p$ and $r=p^{\prime}$. For $r=p$ we observe that the natural map

$$
L_{\mathcal{L}_{E}^{p^{\prime}(\widehat{G})}}^{p}(\Omega) \longrightarrow \mathcal{L}_{L_{E}^{p}(\Omega)}^{p^{\prime}}(\widehat{G})
$$

is a complete contraction. The proof of this fact is similar to that of Theorem 6.1. In particular we have

$$
\begin{aligned}
\left\|\left(\widehat{f}_{i j}\right)\right\|_{S_{n}^{p}\left(\mathcal{L}_{L_{E}^{p^{p}}(\Omega)}(\widehat{G})\right)} & \leq\left\|\left(\widehat{f}_{i j}\right)\right\|_{L_{S_{n}^{p}\left(\mathcal{L}_{E}^{p^{\prime}}(\widehat{G})\right)}(\Omega)} \\
& \leq \mathcal{C}_{p}^{1}(E, G)\left\|\left(f_{i j}\right)\right\|_{S_{n}^{p}\left(L_{L_{E}^{p}(\Omega)}^{p}(G)\right)} .
\end{aligned}
$$

For $r=p^{\prime}$ we use Theorem 6.1] or Lemma 6.4, depending on the measure space $(\Omega, \mathcal{M}, \nu)$, to get the desired relation

$$
\begin{aligned}
& \left\|\left(\widehat{f}_{i j}\right)\right\|_{S_{n}^{p^{p^{\prime}}\left(\mathcal{L}_{L_{E}^{p^{p^{\prime}}(\Omega)}}^{(\widehat{G}))}\right.}} \leq \mathcal{C}_{p}^{1}(E, G)\left\|\left(f_{i j}\right)\right\|_{L_{S_{n}^{p^{p^{\prime}}\left(L_{E}^{p}(G)\right)}}(\Omega)} \\
& \leq \mathcal{C}_{p}^{1}(E, G)\left\|\left(f_{i j}\right)\right\|_{S_{n}^{p^{\prime}\left(L_{L_{E}^{p^{\prime}}(\Omega)}^{p}(G)\right)}} .
\end{aligned}
$$

The proof of the inequality $\mathcal{C}_{q^{\prime}}^{2}\left(L_{E}^{s}(\Omega), G\right) \leq \mathcal{C}_{q^{\prime}}^{2}(E, G)$ is analogous.

Remark 6.6 The proof of Theorem 6.5 for scalar-valued Lebesgue spaces is much simpler. Namely, one only has to see that $L^{2}(\Omega)$ has Fourier type 2 and then the result follows by duality and complex interpolation with the trivial cases $p=1$ and $p=\infty$. But the case $p=2$ is a simple consequence of Plancherel theorem on compact groups.

It is well known that the dual of $L_{E}^{p}(\Omega)$ is not in general $L_{E^{\star}}^{p^{\prime}}(\Omega)$. However it is so when the dual $E^{\star}$ possesses the Radon Nikodym property $R N P$. In 21] Pisier developed an operator space version of the Radon Nikodym property which he called $O R N P$. The following corollary, which is a very simple consequence of Theorems 5.5 and 6.5. shows that both spaces have the same Fourier type and cotype even if $E^{\star}$ does not satisfy the $O R N P$. 
Corollary 6.7 Let $1 \leq p, q \leq 2$ and assume that $E$ is an operator space having Fourier type $p$ and Fourier cotype $q^{\prime}$ with respect to a compact group $G$. Let $(\Omega, \mathcal{M}, \nu)$ be a regular or $\sigma$-finite measure space. Then we have

1. $\mathcal{C}_{q}^{1}\left(L_{E}^{s}(\Omega)^{\star}, G\right)=\mathcal{C}_{q}^{1}\left(L_{E^{\star}}^{s^{\prime}}(\Omega), G\right)$ for all $q \leq s \leq q^{\prime}$.

2. $\mathcal{C}_{p^{\prime}}^{2}\left(L_{E}^{r}(\Omega)^{\star}, G\right)=\mathcal{C}_{p^{\prime}}^{2}\left(L_{E^{\star}}^{r^{\prime}}(\Omega), G\right)$ for all $p \leq r \leq p^{\prime}$.

We now study the Fourier type and cotype of Schatten classes. We will denote by $S^{p}$ the infinite-dimensional Schatten class of exponent $p$. The definition and properties of the vector-valued version of $S^{p}$ are similar to the finite-dimensional case, see Chapter 1 of [21]. We omit the proof of the following result since the arguments to be used can be found in the proof of Theorem 6.5.

Theorem 6.8 Let $1 \leq p, q \leq 2$ and assume that $E$ is an operator space having Fourier type $p$ and Fourier cotype $q^{\prime}$ with respect to a compact group G. Then

1. $S^{r}(E)$ has Fourier type $p$ with respect to $G$ for all $p \leq r \leq p^{\prime}$.

2. $S^{s}(E)$ has Fourier cotype $q^{\prime}$ with respect to $G$ for all $q \leq s \leq q^{\prime}$.

Moreover, $\mathcal{C}_{p}^{1}\left(S^{r}(E), G\right)=\mathcal{C}_{p}^{1}(E, G)$ and $\mathcal{C}_{q^{\prime}}^{2}\left(S^{s}(E), G\right)=\mathcal{C}_{q^{\prime}}^{2}(E, G)$.

Remark 6.9 We already know that the Fourier type and cotype become stronger conditions on the pair $(E, G)$ as $p$ and $p^{\prime}$ approach 2 . This gives rise to the notions of sharp Fourier type and cotype exponents. The problem of finding the sharp exponents of a given operator space is highly non-trivial even for the simplest case of Lebesgue spaces or Schatten classes. Part of this problem is solved in [12]. Namely, if $1 \leq p \leq 2$ and $(\Omega, \mathcal{M}, \nu)$ is not the union of finitely many $\nu$-atoms, then we show that $L^{p}(\Omega)$ has sharp Fourier type $p$ with respect to any compact semisimple Lie group. By duality we also get that $L^{p^{\prime}}(\Omega)$ has sharp Fourier cotype $p^{\prime}$ for those groups. By the nature of $\Omega$ and Proposition 5.2 we have

$$
\mathcal{C}_{q}^{1}\left(L^{p}(\Omega), G\right) \geq \lim _{n \rightarrow \infty} \mathcal{C}_{q}^{1}\left(l^{p}(n), G\right)
$$

for $1 \leq p<q \leq 2$. Moreover, Theorem 5.8 gives $\mathcal{C}_{q}^{1}\left(l^{p}(n), G\right) \leq n^{1 / p-1 / q}$. The main result of 12 asserts that there exists a positive constant $\mathcal{K}(G, q)$, 
such that $\mathcal{K}(G, q) n^{1 / p-1 / q} \leq \mathcal{C}_{q}^{1}\left(l^{p}(n), G\right) \leq n^{1 / p-1 / q}$ for all $n \geq 1$ and any compact semisimple $G$. The constant $\mathcal{K}(G, q)$ can be defined as

$$
\mathcal{K}(G, q)=\inf _{n \geq 1} \sup \left\{\frac{\|\widehat{f}\|_{\mathcal{L}^{q^{\prime}}(\widehat{G})}}{\|f\|_{L^{q}(G)}}: \quad f \quad \text { central, } \quad f \in L^{q}(G), \quad \operatorname{supp}(f) \subset \mathcal{U}_{n}\right\}
$$

where $\left\{\mathcal{U}_{n}: n \geq 1\right\}$ denotes a neighborhood basis at the identity of $G$. The interesting point lies in the inequality $\mathcal{K}(G, q)>0$ which constitutes a local variant of the Hausdorff-Young inequality on $G$ with parameter $q$. The proof obtained for this local inequality is based upon the semisimplicity of $G$ since it uses the very well-developed theory of representations on such kind of groups. The need to use these algebraic techniques forced us to present the proof of this result in a separate work, see [12].

\section{References}

[1] M.E. Andersson, 'On the vector valued Hausdorff-Young inequality', Ark. Mat. 36 (1998) 1-30.

[2] D.P. Blecher And V.I. Paulsen, 'Tensor Products of Operator Spaces', J. Funct. Anal. 99 (1991) 262-292.

[3] J. Bourgain, 'A Hausdorff-Young inequality for B-convex Banach spaces', Pacific J. Math. 101 (1982) 255-262.

[4] J. Bourgain, 'Vector-valued Hausdorff-Young inequality and applications', Geometric aspects of functional analysis (eds J. Lindenstrauss and V.D. Milman), Lecture Notes in Mathematics 1317 (Springer, Berlin, 1988), pp. 239-249.

[5] D.L. Cohn, Measure Theory (Birkhäuser, Boston, 1980).

[6] J. Dixmier, 'Formes linéaires sur un anneau d'operateurs', Bull. Soc. Math. France 81 (1953) 9-39.

[7] E.G. Effros and Z.J. Ruan, 'A New Approach to Operator Spaces', Canad. Math. Bull. 34 (1991) 329-337.

[8] E.G. Effros And Z.J. Ruan, Operator Spaces, London Math. Soc. Monogr. 23, (Oxford Univ. Press, New York, 2000). 
[9] G.B. Folland, A Course in Abstract Harmonic Analysis, Stud. Adv. Math. (CRC Press, Boca Raton, 1995).

[10] J. García-Cuerva, K. Kazarian, V. Kolyada and J.L. Torrea, 'Vector-valued Hausdorff-Young inequality and applications', Russian Math. Surveys (3) 53 (1998) 435-513.

[11] J. García-Cuerva, K. Kazarian and V. Kolyada, 'Paley type inequalities for orthogonal series with vector-valued coefficients', Acta Mat. Hungarica 90 (2001) 151-183.

[12] J. García-Cuerva, J.M. Marco and J. Parcet, 'Sharp Fourier type and cotype with respect to compact semisimple Lie groups'Trans. Amer. Math. Soc. 355 (2003), 3591-3609.

[13] J. García-Cuerva And J. PArCet, 'Quantized orthonormal systems: A non-commutative Kwapień theorem', Studia Math. 155 (2003) 273294.

[14] H. KÖNIG, 'On Fourier coefficients of vector-valued functions', Math. Nachr. 152 (1991) 215-227.

[15] R.A. Kunze, ' $L_{p}$ Fourier transforms on locally compact unimodular groups', Trans. Amer. Math Soc. 89 (1958) 519-540.

[16] S. KWAPIEŃ, 'Isomorphic characterizations of inner product spaces by orthogonal series with vector valued coefficients', Studia Math. 44 (1972) 583-595.

[17] M. Milman, 'Complex interpolation and geometry of Banach spaces', Ann. Mat. Pura Appl. 136 (1984) 317-328.

[18] J. PARCET, Análisis armónico no conmutativo y geometría de espacios de operadores. Ph.D. Thesis (2003).

[19] J. Peetre, 'Sur la trasnformation de Fourier des fonctions à valeurs vectorielles', Rend. Sem. Mat., Univ. Padova, 42 (1969) 15-26.

[20] G. PIsIER, 'The Operator Hilbert Space OH, Complex Interpolation and Tensor Norms', Mem. Amer. Math. Soc. 122 (1996) 1-103. 
[21] G. Pisier, 'Non-Commutative Vector Valued $L_{p}$-Spaces and Completely p-Summing Maps', Astérisque (Soc. Math. France) 247 (1998) $1-111$.

[22] Z.J. Ruan, 'Subspaces of $C^{\star}$-algebras', J. Funct. Anal. 76 (1988) 217230.

[23] I.E. SEgal, 'An extension of Plancherel's formula to separable unimodular groups', Ann. of Math. 52 (1950) 272-292.

[24] I.E. SEgal, 'A noncommutative extension of abstract integration', Ann. of Math. 57 (1953) 401-457.

José García-Cuerva and Javier Parcet

Departamento de Matemáticas, C-XV, Universidad Autónoma de Madrid, 28049 Madrid, Spain

jose.garcia-cuerva@uam.es

javier.parcet@uam.es 